BHL 51536

UC-28

IParticle Accelerators and

High-Voltage Machines - TIC-4500)

ISA 82-3

BHL -51536

DE82 020553

\title{
A STUDY ON THE CONDITIONS REQUIRED FOR THE TRANSVERSE STABILITY \\ OF A COASTING BEAM IN PROTON STORAGE RINGS
}

M. Cornacchia and C. Pellegrini

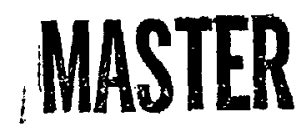

February 1982

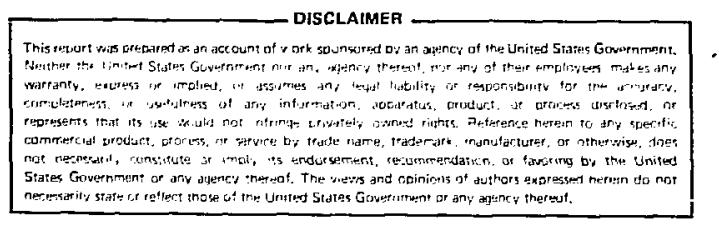

ACCELERATOR DEPARTMENT

BROOKHAVEN NATIONAL LABORATORY

UPTON, LONG ISLAND, NEW YORK 11973 
CONTENTS

INTRODUCTION.

\section{1}

PART I THEORETICAL DESCRIPTION OF THE TRANSVERSE INSTABILITY.......... 2

1. The Equation of the Transverse Oscillations................ 2

2. The Dispersion Relation........................... 6

3. The Coherent and Incoherent Tune Shifts.....................

4. Space-Charge Effect on Threshold Currert: A Simple Mode1........12

PART II CONDITIONS FOR TRANSVERSE STABILITY IN ISABELLE............. 20

1: Numerical Integration of the Dispersion Relation..............20

2. Fully Stacked Debunched Beam at the Center of the Chamber.......21

3. Bunched Beam at the Center of the Chamber.....................

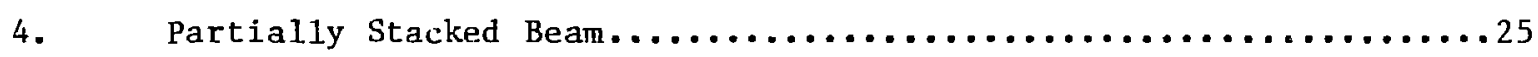

5. Fully stacked Beam, off-Axis........................ 28

6. Partially Neutralized High Energy Beam................... 28

7. Discussion on the Beam Dynamics Assumptions and Impedance Mode1...29

8. Discussions of Space-Charge Effects in the ISR and ISABELLE......30

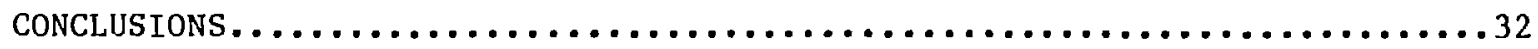

APPENDIX A. Coherent and Incoherent Space-Charge Tune Shifts in ISABELLE..33

APPEndix B. Transverse Space-Charge Distribution Function...............36

APPENDIX $c$. Impedance of an Off-Axis Beam......................... 


\section{ABSTRACT}

A general theory of the transverse instability of coasting beams in circular accelerators produced by the interaction of the beam charge and current with its electromagnetic environment is presented. The theory allows to numerically calculate the threshold current for an arbitrary frequency versus momentum curve. The numerical solution is used to study the stability of a coasting beam in a high energy proton storage ring like ISABELLE during the stacking process and for a full intensity beam. 


\section{INTRODUCTION}

A coasting beam in a circular accelerator or storage ring can execute unstable transverse coherent oscillations produced by the interaction of the beam charge and current with its electromagnetic environment, l usually described as the accelerator impedance. If the particles in the beam have different betatron oscillation frequencies, a "Landau damping" of the coherent oscillation is produced. When Landau damping is present the instability will occur only when the beam eurrent is larger than a "threshold current."

In the simplest case the variation in betatron frequency is due to the machine chromaticity (change in frequency with momentum), and the frequency depends linearly on momentum. In this case the tnreshold current is proportional to the frequency spread in the beam.

Nonlinear force terms, produced by the beam space-charge effects or by the guiding magnetic field, can introduce a nonlinear dependence of the oscillation frequency on the particle momentum. Observations at $\operatorname{ISR}^{2}$ have shown that the threshold current value depends strongly on the shape of the frequency versus momentum curve. In particular the beam can become unstable, also for a large total frequency spread, if the derivative of the betatron frequency with respect to the momentum becomes sma11.2 In other words, if the local frequency spread, described by the derivative of the frequency with respect to the momentum, becomes zero or nearly zero, the beam may become unstable. In fact, by "shaping the working line", i.e., the frequency versus momentum curve, the stable current in the ISR has been raised by more than one order of magnitude. This is done by using many correcting coils that can control the magnetic field locally and thus allow one to obtain the "best" frequency versus momentum curve.

A calculation of the change in the threshold value produced by a parabolic distribution of the frequency due to space-charge effects has been done by Month and Jellet. ${ }^{3}$ In their work the strength of the space-charge perturbation is also limited, so that the derivative of the oscillation frequency with respect to the momentum does not change sign. Their results are in qualitative agreement with the ISR result and confirm the physical interpretation that the beam is unstable if the local frequency spread is too small.

1. L.J. Laslett et al., Rev. Sc1. Instrum. 36, 436 (1965).

2. See, e.g., W. Schne11, Report on the ISR, IEEE Trans. Nucl. Sc1. NS-22, 1358 (1975), where references to other reports can also be found.

3. M. Month and K. Jellet, Nucl. Instrum. Methods 113, 453 (1973). 
In this work we develop a more general theory that allows calculation of the threshold current for an arbitrary frequency versus momentum curve. This theory is first applied to a simple case in which an analytical solution of the stability problem can be obtained. This simple case describes the effect of a parabolic term in the frequency-momentum curve, and it shows that the threshold current depends not only on the magnitude of the quadratic term but also on the relative sign of the linear and quadratic terms. In fact one sign leads to an increase of the threshold current when the quadratic term increases, and the other to a decrease.

The general case of an arbitrary frequency distribution is then solved numerically. This numerical solution is used to study the stability of a coasting beam in a high energy proton storage ring like ISABELLE during the stacking process and for a full intensity beam. This study is done for different momentum distribution curves and includes the effect of the nonlinear space-charge term and of up to sixteen independent magnetic multipoles. The algorit?m that has been developed has allowed calculation of both the number and the strength of the magnetic multipoles needed to provide a stable beam of the desired current.

\section{PART I}

THEORETICAL DESCRIPTION OF THE TRANSVERSE INSTABILITY

\section{The Equation of Transverse Oscillations}

In this section we introduce the Hamiltonian describing the transverse motion of a coasting beam. We introduce explicitly the nonlinear force terms due to the space-charge forces or to the magnetic field. We assume the beam to be moving in one transverse dimension only and include the longitudinal direction, neglecting the coupling between the radial and vertical oscillation modes.

We use the term y for the transverse direction, $\phi$ for the azimuthal angle in the beam rotating frame, and $\varepsilon$ for the relative energy displacement with respect to a reference particle energy, $E_{0}$.

The beam motion in the $y, \dot{y}$ plane can be obtaineu from the Hamiltonian ${ }^{4}$

$$
\begin{aligned}
H= & \frac{1}{2} \dot{y}^{2}+\frac{1}{2} v_{o}^{2} \omega_{o}^{2}(1+2 P \varepsilon) y^{2} \\
& -\frac{1}{2} \omega_{o} \eta \varepsilon^{2}+H_{N L}-F y .
\end{aligned}
$$

We use the smooth approximation to describe the transverse oscillations.

4. See, e.g., C. Pellegrini, Single beam coherent instabilities in circular accelerator and storage rings, in Proc. 1981 Summer school on Particle Accelerators, Amer. Inst. Phys. Conf. Series, 1982. 
In Eq. (1) $\nu_{0}$ is the betatron tune, $\omega_{0}$ is the revolution frequency, and $\eta$ is the frequency slip factor

$$
\eta=\frac{1}{\gamma_{T}^{2}}-\frac{1}{\gamma_{0}^{2}}=-\frac{1}{\omega_{0}} \frac{\partial \omega_{o}}{\partial \varepsilon}
$$

with $\gamma_{0}$ the energy in rest mass units, $\gamma_{0}=E_{0} / \mathrm{mc}^{2}$, and $\gamma_{T}$ the transition energy; $P$ is related to $\eta$ and the chromaticity

$$
\xi=\frac{1}{v_{0}} \frac{d \nu_{0}}{d \varepsilon}
$$

by

$$
P=\xi-\eta
$$

The term $\mathrm{H}_{\mathrm{NL}}$ in $\mathrm{Eq}$. (1) describes the nonlinear forces acting on a particle, and the term Fy the collective forces.

Nonlinear forces are produced by nonlinear terms in the machine magnetic field. The force in the $y$ direction is produced by the field in the orthogonal direction, $x$, which we write in the form

$$
\frac{e \beta B}{m} x=\omega_{0}^{2} \sum_{n=2}^{N} \sum_{k=0}^{n} b_{n}, k^{x^{n-k} y^{k}}
$$

where $\mathrm{B}_{\mathrm{O}}$ is the guide magnetic field. Using $\mathrm{Eq}$. (5) we can write the Hamiltonian term, $\mathrm{H}_{\mathrm{NL}}$, as

$$
\mathrm{H}_{\mathrm{NL}}=-\omega_{0}^{2} \sum_{\mathrm{n}=2}^{\mathrm{N}} \sum_{\mathrm{k}=0}^{\mathrm{n}} \mathrm{b}_{\mathrm{n}, \mathrm{k}}^{\mathrm{k}+\mathrm{I}} \mathrm{x}^{\mathrm{n}-\mathrm{k}_{\mathrm{y}} \mathrm{k}+\mathrm{l}} .
$$

Since we are interested mainly in the frequency spread produced by the nonlinear forces, we can identify some important terms in $\mathrm{Eq}$. (6). One term is the octupcle term, $\mathrm{n}=\mathrm{k}=3$. Another contribution is produced by the energy spread. Assuming $x=x+x_{p} \varepsilon$, we can identify, for vertical oscillations, a term $k=1, n$ arbitrary. In the case of radial oscillation we take $\mathrm{n}=\mathrm{k}$ and $\mathrm{y}^{\mathrm{k}+1}=\mathrm{y}^{2}\left(\mathrm{X}_{\mathrm{p}} \varepsilon\right)^{\mathrm{k}-1}$.

If we choose the $v_{0}$ value such that nonlinear resonances are avoided, we can neglect all terms in Eq. (6) sxcept those producing a frequency spread, and can write, in the case of vertical oscillations,

$$
\mathrm{H}_{\mathrm{NL}}=-\omega_{\mathrm{o}}^{2}\left\{\frac{\mathrm{b}_{3}, 3}{4} \mathrm{y}^{4}+\frac{\mathrm{y}^{2}}{2} \sum_{\mathrm{n}=2}^{\mathrm{N}} \mathrm{b}_{\mathrm{n}, 1}\left(\mathrm{x}_{\mathrm{p}} \varepsilon\right)^{\mathrm{n}-1}\right\}
$$


and, for radial oscillations,

$$
\mathrm{H}_{\mathrm{NL}}=-w_{0}^{2}\left\{\frac{1}{4} b_{3,3^{4}} \mathrm{y}^{4}+y^{2} \sum_{n=2}^{N} \frac{b_{n, n}}{n+1}\left(x_{p} \varepsilon\right)^{n-1}\right\} \text {. }
$$

To take into account the AGS structure of the machine, we can substitute in Eqs. (7) and (8) the term $b_{n} x_{p}^{n-1}$ with

$$
b_{n} x_{p}^{n-1}+\frac{\nu_{0}}{2 \pi R^{2}} \int_{0}^{2 \pi R} b_{n} x_{p}^{n-1} \beta d s
$$

where $b_{n}$ stands for $b_{n, l}$ or $b_{n, n}$ and $\beta$ is the horizontal or vertical $\beta$ function.

The collective force produces a coherent and an incoherent term. We consider all this term to be linear except for the incoherent space-charge term. We can incorporate the linear part of the incoherent tune shift in $\nu_{0}$, to first order, and neglect it. The norlinear part produces a frequency spread and we consider it explicitly.

We write the incoherent space-charge Hamiltonian as

$$
H_{s c}=2 v_{0} \Delta v_{s c} \frac{\omega_{o}^{2}}{S_{1,1}} \sum_{n, k} \frac{S_{n, k}}{k+I} x^{n-k} y^{k+1}
$$

and assume, as done previously, for the vertical case, $k=1, x=x_{p} \varepsilon$ or $\mathrm{k}=3, \mathrm{n}=\mathrm{k}$. For the vertical oscillation case we then have

$$
H_{s c}=2 v_{0} \frac{\Delta v_{s c}}{s_{1,1}} \omega_{o}^{2} y^{2}\left\{\frac{s_{3,3}}{4} y^{2}+\sum_{n} \frac{s_{n, 1}}{2}\left(x_{p} \varepsilon\right)^{n-1}\right\} \text {. }
$$

For the radial oscillation case we have

$$
H_{s c}=2 v_{0} \frac{\Delta v_{s c}}{S_{1,1}} \omega_{c}^{2} y^{2}\left\{\frac{s_{3}, 3}{4} y^{2}+\sum_{n} \frac{s_{n, n}}{n+1}\left(x_{p} \varepsilon\right)^{n-1}\right\} .
$$

The coefficients $S_{n, k}$ depend on the beam geometry. For a uniform cylindrical beam in a vacuum or on the axis of a cylindrical vacuum chamber we have

$$
\Delta v_{s c}=-\frac{e I_{o} Z_{o} R^{2}}{4 \pi v_{0} \beta E}\left(\frac{1}{\beta^{2} \gamma^{2}}-f\right) \frac{1}{a^{2}}
$$

where $Z_{0}$ is the vacuum impedance, $f$ is the fractional beam neutralization, and $a$ is the beam radius. In this case all the other coefficients $s_{n, k}$ with $\mathrm{n}>\mathrm{l}$ are zero. 
The values of the $S_{n, k}$ that can be used to describe ISABELLE will be discussed later on.

To write explicitly the coherent collective force, we need to introduce the particle distribution function, which we write as ${ }^{4}$

$$
f=\frac{N}{R}\left\{\frac{1}{2 \pi} g_{o}(\varepsilon) h_{o}(y, \dot{y})+f_{1}^{P}(y, \dot{y}, \varepsilon) e^{i p \phi-i v \omega_{o} t}\right\}
$$

which describes an unstable motion when the imaginary part of the coherent tune, $\nu$, is positive:

$$
\text { Im } v>0 \text { unstable. }
$$

In $E_{q}$. (14) the term $g_{o}(\varepsilon) h_{o}(y, y)$ describes the equilibrium solution, and the term $\mathrm{f}_{1 \mathrm{p}}$ the perturbation around the equilibrium. The perturbation is periodic along the ring, and $p$ is the number of waves in the perturbation.

Using Eq. (14) we obtain for $\mathrm{F}$ the expression ${ }^{4}$

$$
F=\frac{i e I_{0} R \omega_{0}^{2}}{2 \pi \beta E} D_{p} Z_{\perp}\left(p+v_{o}\right) e^{i p \phi-i v \omega_{o} t}
$$

where $D_{p}$ is the beam dipole moment

$$
\mathrm{D}_{\mathrm{p}}=\int \mathrm{yf}{ }_{1}(\mathrm{y}, \dot{\mathrm{y}}, \varepsilon) \mathrm{d} \mathrm{yd} \dot{\mathrm{y}} \mathrm{d} \varepsilon
$$

and $z_{1}\left(p+v_{0}\right)$ is the storage ring transverse impedance evaluated at the frequency $\omega=\left(p+\nu_{0}\right) \omega_{0}$.

The transverse impedance is assumed to be given by the space-charge term, a term due to a perfectly conducting wall, a term describing the effect of the wall resistivity, and a term describing the high frequency effects as given by the broad-band impedance. ${ }^{4}$ For a uniform cylindrical beam on the axis of a cylindrical vacuum pipe of radius $b$, we have ${ }^{4}$

$$
\begin{aligned}
& Z_{L}(\omega)= i Z_{o} R\left\{\frac{2}{\beta^{2} \gamma^{2}}\left(\frac{1}{a^{2}}-\frac{1}{b^{2}}\right)\right. \\
&\left.-(1+i) \frac{\delta(\omega)}{b^{3}}-\frac{2 i}{b^{2}} \frac{Z_{\|}^{B}(\omega)}{\omega} \frac{\omega_{O}}{Z_{o}}\right\} \\
& Z_{\|}^{B}(\omega)=R_{S} \frac{\omega}{\omega_{R}} \frac{\left(\omega / \omega_{R}\right)-i Q\left[1-\left(\omega / \omega_{R}\right)^{2}\right]}{\left(\omega / \omega_{R}\right)^{2}+Q^{2}\left[1-\left(\omega / \omega_{R}\right)^{2}\right]^{2}}
\end{aligned}
$$

and

$$
\omega_{R} \approx C /(2 b), Q \approx 1, K_{S}=\text { shunt impedance. }
$$


The skin depth $\delta(\omega)$ is given, for a metal of conductivity $\sigma$, by

$$
\delta(\omega)=c /(2 \pi \omega \sigma)^{1 / 2}
$$

for $\omega>0$.

The impedance $z_{\perp}(\omega)$ must satisfy the conditions ${ }^{4}$

$$
\operatorname{Re} z_{1}(-\omega)=-\operatorname{Re} Z_{1}(\omega)
$$

and

$$
\operatorname{Im} z_{\perp}(-\omega)=\operatorname{Im} z_{\perp}(\omega)
$$

which requires that

$$
\delta(-\omega)=-i \delta(|\omega|)
$$

The case of a beam which is displaced with respect to the axis of the vacuum chamber, as happens during stacking in ISABELLE, is discussed in Appendix $C$.

2. The Dispersion Relation

Using the results of the previous section we rewrite the Hamiltonian Eq. (1), in the vertical case, as

$$
\begin{aligned}
H & =\frac{1}{2} \dot{y}^{2}+\frac{1}{2} v_{o}^{2} \omega_{o}^{2} y^{2}\left\{1+2 P_{\varepsilon}-\frac{5_{3,3}}{2} y^{2}\right. \\
& \left.-\sum_{n=2}^{N} 5_{n, 1}\left(X_{p} \varepsilon\right)^{n-1}\right\}-\frac{1}{2} \omega_{o} \eta \varepsilon^{2}-F y
\end{aligned}
$$

where

$$
\bar{b}_{3,3}=\frac{b_{3,3}}{v_{0}^{2}}-\frac{2}{v_{0}} \Delta v_{\text {sc }} \frac{s_{3,3}}{s_{1,1}}
$$

and

$$
\overrightarrow{\mathrm{b}}_{\mathrm{n}, 1}=\frac{\mathrm{b}_{\mathrm{n}, 1}}{v_{\mathrm{o}}^{2}}-\frac{\Delta v_{\mathrm{sc}}}{v_{\mathrm{o}}} \frac{\mathrm{s}_{\mathrm{n}, 1}}{\mathrm{~s}_{1,1}}
$$

and obtain a similar expression for the radial case. 
We now introduce action-angle varlables, I, $\Psi$, such that

$$
\begin{aligned}
& y=\left(\frac{2 I}{v_{0} \omega_{0}}\right)^{1 / 2} \cos \Psi, \\
& \dot{y}=-\left(2 \nu_{0} \omega_{0} I\right)^{1 / 2} \sin \Psi
\end{aligned}
$$

to obtain the Hamiltonian

$$
\begin{aligned}
H= & v_{0} \omega_{0} I\left\{1+P \varepsilon-\frac{1}{2} \sum_{n=2}^{N} \bar{b}_{n, 1}\left(X_{p} \varepsilon\right)^{n-1}-\frac{3}{8} \frac{\bar{b}_{3,3}}{v_{o} \omega_{o}} I\right\} \\
& -\frac{1}{2} \omega_{0} n \varepsilon^{2}-F\left(\frac{2 I}{v_{0} \omega_{0}}\right)^{1 / 2} \cos \Psi
\end{aligned}
$$

where we have neglected all fast oscillating terms.

We can now use the Hamiltonian Eq. (29) and the linearized Vlasov equation to obtain the dispersion relation ${ }^{4}$

$$
1=-\delta v_{p} \int d \varepsilon d I \frac{I \frac{\partial h_{o}(I)}{\partial I} g_{0}(\varepsilon)}{\nu-v_{0}+\frac{3}{4} \frac{\bar{b}_{3,3}}{\omega_{0}} I-v_{0}\left[\left(P-\frac{n p}{v_{0}}\right) \varepsilon-\frac{1}{2} \sum_{n=2}^{N} b_{n, 1}\left(x_{p} \varepsilon\right)^{n-1}\right]}
$$

with the coherent tune shift $\delta v_{p}$ given by

$$
\delta v_{p}=\frac{-i e I_{o} R}{4 \pi \beta E_{0} v_{o}} z_{\perp}\left(p+v_{0}\right)
$$

The dispersion relation Eq. (30) is rather complicated because of its dependence on both the energy and the betatron amplitude distribution. We are interested in obtaining from Eq. (30) a condition for beam stability. Both the amplitude and the energy spread produce Landau damping and stabilize the beam. Hence, if we consider only one of these two effects, we obtain a stronger limitation on the beam current than in the case when both are present.

Let us consider the simple case when the frequency spread produced by the change in betatron amplitude is small compared with that produced by the energy distribution. We can neglect the term $\bar{b}_{3,3^{I}}$ in the denominator of $\mathrm{Eq}$. (30) and integrate over I to obtain

$$
1=\delta v_{p} \int d \varepsilon \frac{g_{0}(\varepsilon)}{\nu-v_{0}-v_{o}\left[\left(P-\frac{\eta p}{v_{0}}\right) \varepsilon-\frac{1}{2} \sum_{n=2}^{N} 5_{n, 1}\left(x_{p} \varepsilon\right)^{n-1}\right]} \text {. }
$$


For zero energy spread we obtain the simple solution

$$
v-v_{0}=\delta v_{p}
$$

giving instability if

$$
\text { In } \delta v_{p}>0
$$

i.e., if

$$
\operatorname{Re} z_{1}\left(p+v_{0}\right)<0 .
$$

From. Eq. (17) we have

$$
\operatorname{Re} z_{1}(\omega)>0 \quad \text { if } \quad \omega>0
$$

and

$$
\operatorname{Re} Z_{\perp}(\omega)<0 \text { if } \omega<0 \text {; }
$$

thence the motion is unstable if

$$
p+v_{0}<0
$$

and we have to consider only the values of p satisfying this condition. Since the oscillation frequency in the laboratory frame is $\left(p+v_{0}\right) \omega_{0}$, condition $\mathrm{Eq}$. (38) is equivalent to requiring that only the slow wave be unstable.

Let us now consider the case when the energy spread is important. We rewrite Eq. (32) as

$$
1=\delta v_{p} \int d \varepsilon \frac{g_{0}(\varepsilon)}{\sum_{n=1}^{N} a_{n} \varepsilon^{n-1}}
$$

with

$$
\begin{gathered}
a_{1}=v-v_{0}, \\
a_{2}=-v_{0}\left[p-\frac{n p}{v_{0}}-\frac{1}{2} b_{2,1} x_{p}\right], \\
a_{n}=\frac{v_{0}}{2} b_{n, 1} x_{p}^{n-1} .
\end{gathered}
$$

We want to find a solution of Eq- (39), for $\operatorname{Im} \delta v_{p}>0$, such that $a_{1}$ is real, giving a stable beam. 
In the simple case when

$$
a_{n}=0 \quad \text { for } \quad n>2
$$

the dispersion relation becomes

$$
1=\delta v_{p} \int d \varepsilon \frac{g_{o}(\varepsilon)}{a_{1}+a_{2} \varepsilon}
$$

which we rewrite as

$$
1=\frac{\delta v_{p}}{C_{0}} \int d \varepsilon \frac{g_{o}(\varepsilon)}{\Sigma-\varepsilon}
$$

with

$$
\varepsilon_{1}=-\frac{a_{1}}{a_{2}}, c_{o}=\frac{a_{1}}{-\varepsilon_{1}}=a_{2}, a_{2}=-\left[\nu_{0} \xi-\left(\nu_{0}+p\right) \eta\right] .
$$

For $\xi>0$ we have $a_{2}<0$. If we look for a solution such that

$$
\operatorname{In} v \rightarrow 0^{+}
$$

i.e.,

$$
\operatorname{Im} \varepsilon_{1} \rightarrow 0^{+}
$$

we have, ${ }^{4}$ for $a_{2}<0$,

$$
\int \mathrm{d} \varepsilon \frac{\mathrm{g}_{\mathrm{o}}(\varepsilon)}{\varepsilon-\varepsilon_{1}}=\mathrm{P} \int \mathrm{d} \varepsilon \frac{\mathrm{g}_{\mathrm{o}}(\varepsilon)}{\varepsilon-\varepsilon_{1}}+\mathrm{i} \pi \mathrm{g}_{0}\left(\varepsilon_{1}\right) .
$$

Substituting into Eq. (45) we obtain two equations in two unknown: $a_{1}$, i.e., $v$; and $\delta v_{p}$, i.e., the beam current for which the beam is stable or threshold current. To write this more explicitly let us change notations and define

$$
\begin{gathered}
\frac{1}{C_{0}} \int \mathrm{d} \varepsilon \frac{g_{0}(\varepsilon)}{\varepsilon-\varepsilon_{1}}=Q+i P, P>0 ; \\
\delta \nu_{p}=I_{0}(A+i B), B>0 .
\end{gathered}
$$


We can now rewrite Eq. (45) as

$$
\begin{aligned}
& I_{0} Q=\frac{A}{A^{2}+B^{2}}, \\
& I_{0} P=\frac{-B}{A^{2}+B^{2}},
\end{aligned}
$$

and solve this equation for $I_{0}, a_{1}$. An alternative way is to calculate, as a function of $a_{1}$, the two currents

$$
\begin{aligned}
& I_{O Q}=\frac{1}{Q} \frac{A}{A^{2}+B^{2}}, \\
& I_{O P}=-\frac{1}{P} \frac{B}{A^{2}+B^{2}} .
\end{aligned}
$$

The threshoid curreni is obtained for $I_{O Q}=I_{O P}$. This mechod will be used in the numerical evaluation of the stability limit.

We can follow a similar procedure for the general case. Let $\varepsilon_{1}, \ldots, \varepsilon_{N-1}$ be the roots of the equation

$$
\sum_{n=1}^{N} a_{n} \varepsilon^{n-1}=0
$$

We can write

$$
\sum_{n=1}^{N-1} a_{n} E^{n-1}=C_{0} \underset{n=1}{N-1}\left(\varepsilon-\varepsilon_{n}\right)
$$

where

$$
C_{0}=a_{1} /\left[\prod_{n=1}^{N-1}\left(-\varepsilon_{n}\right)\right]
$$

The integral in Ea. (39) can now be written as

$$
\int d \varepsilon \frac{g_{o}(\varepsilon)}{\sum_{n=1}^{N} a_{n} \varepsilon^{n-1}}=\frac{1}{C_{0}} \sum_{n=1}^{N-1} \int d \varepsilon \frac{C_{n} g_{o}(\varepsilon)}{\varepsilon-\varepsilon_{n}}
$$

where

$$
C_{m}^{-1}=\underset{H \neq h}{N-1}\left(\varepsilon_{m}-\varepsilon_{n}\right)
$$


For $a_{1}$ real $c_{o}$ also is real, since the $\varepsilon_{n}$ are either real or complex conjugate. For a couple of complex conjugate roots the integral has no singularí, , and for $v$ and $a_{1}$ real the corresponding integrals in Eq. (59) are also real. For zeal roots we can follow the same procedure given for the case of $a_{n}=0$ for $n>2$, and the integral has both a real and an imaginary part. If we again define as in Eq. (50),

$$
\frac{1}{C_{o}} \sum_{n=1}^{N} C_{n} \int d \varepsilon \frac{g_{o}(\varepsilon)}{\varepsilon-\varepsilon_{n}}=Q+i P
$$

we can evaluate from $I_{o Q}, I_{o P}$ Eqs. (54) and (55) and obtain the threshold current for the value of $a_{1}$ such that $I_{O Q}=I_{O P}=I_{t h}$.

3. The Coherent and Incoherent Tune Shift

To evaluate the dispersion relation and obtain a threshold current we need firsi to establish the value of the coherent and incoherent tune shifts. In this section we do this by using the parameters and a model of the beam geometry which apply to the ISABELLE storage ring.

From Eqs. (17) and (31) we obtain

$$
\begin{gathered}
\delta v_{p}=\frac{e I_{o} Z_{o}}{4 \pi \beta E_{o} v_{o}}\left(\frac{R}{b}\right)^{2}\left\{\frac { 1 } { \beta ^ { 2 } \gamma ^ { 2 } } ( 1 - f ) \left(\frac{b^{2}}{\left.a^{2}-1\right)}\right.\right. \\
-(1-i) \frac{\delta\left(\omega_{o}\right)}{b} \frac{1}{\sqrt{T}+v_{o} T} \\
-2 \frac{R_{S}}{n_{R_{o}^{2}}} \frac{Q\left[1-\left(\frac{p+v_{o}}{n_{R}}\right)^{2}\right]-i\left|\frac{p+v_{o}}{n_{R}}\right|}{\left.P-\left(\frac{p+v_{o}}{n_{R}}\right)^{2}\right]^{2}+\left(\frac{p+v_{o}}{n_{R}}\right)^{2}} \\
P+v_{o}<0, \\
\text { Im } \delta v_{p}>0 .
\end{gathered}
$$

To evaluate Eq. (62) for ISABELIE we assume, for a beam which is at the center of the vacuum chamber,

$$
\mathrm{b}=4.4 \mathrm{~cm}, \quad \delta\left(\omega_{\mathrm{o}}\right)=1.7 \mathrm{~mm}, \frac{\mathrm{R}_{\mathrm{s}}}{\mathrm{n}_{\mathrm{R}}}=10 \Omega, \quad \mathrm{Q}=1, \quad \mathrm{n}_{\mathrm{R}}=7000 .
$$


For the space-charge coherent term we assume for the vertical case (see Appendix A)

$$
\begin{array}{llrl}
\delta \nu_{\text {coh }}=1.6 \times 10^{-3} \mathrm{I}_{\mathrm{o}} & \text { at } & 30 \mathrm{GeV}, \mathrm{f}=0 ; \\
\delta \nu_{\text {coh }}=-4.6 \times 10^{-4} \mathrm{I}_{\mathrm{o}} & \text { at } & 400 \mathrm{GeV}, \mathrm{f}=2 \times 10^{-4} ;
\end{array}
$$

and for the horizontal case

$$
\delta v_{\mathrm{coh}}=1.6 \times 10^{-3} \mathrm{I}_{\mathrm{o}} \text { at } 3 \mathrm{G}_{\mathrm{GeV}} \mathrm{f}=0 .
$$

For the incoherent term we assume in the vertical case at $30 \mathrm{GeV}, \mathrm{f}=0$.

$$
\delta v_{\text {inc }}=-1.6 \times 10^{-3} \mathrm{I}_{\mathrm{o}}+3 \times 10^{-3}\left(\frac{\varepsilon}{\varepsilon_{\mathrm{M}}}\right)^{2} \text { at } 30 \mathrm{GeV}, \mathrm{f}=0
$$

where $\varepsilon_{M}$ is the maximum momentum deviation in the beam.

In Figure 1 the various impedance terms appearing in Eq. (62) are plotted versus $n=-p$. The quantity $\delta v S C$ is the space-charge term, the first term on the right side of $\mathrm{Eq},(62), \delta \nu^{\mathrm{RW}}$ is the second term, and $\delta v^{\mathrm{BB}}$ is the last term. The coherent tune shift refers to the sases where the beam is centered in the vacuum chamber and where the heam is displaced by an average $2 \mathrm{~cm}$ off axis in the region of the ring where the dispersion is not zero. This latter case corresponds to a momentum stacked beam, $20 \%$ of the total current, with an average moment'm offset $\varepsilon_{d}=0.005$. How the coherent tune shift of Eq. (62) is modified by this displacement i's discussed in Appendix $c$.

4. Space Charge Effect on Threshold Current: A Simple Model

In this section we want to estimate the affect of the octupolar spacecharge term in Eq. (68) on the current threshold using a simple model, i.e., a parabolic energy distribution and only a sextupolar magnetic field term. This means that we use only a term $a_{2}$ given by Eq. (46)

$$
a_{2}=\left(\nu_{0}+p\right) \eta-\nu_{0} \xi
$$

and a term $a_{3}$ corresponding to the last term in Eq. (68).

Let us first consider the case when $a_{3}=0$ also. Assuming

$$
g_{0}(\varepsilon)=\frac{3}{4 \varepsilon_{M}}\left\{1-\left(\frac{\varepsilon}{\varepsilon_{M}}\right)^{2}\right\}
$$




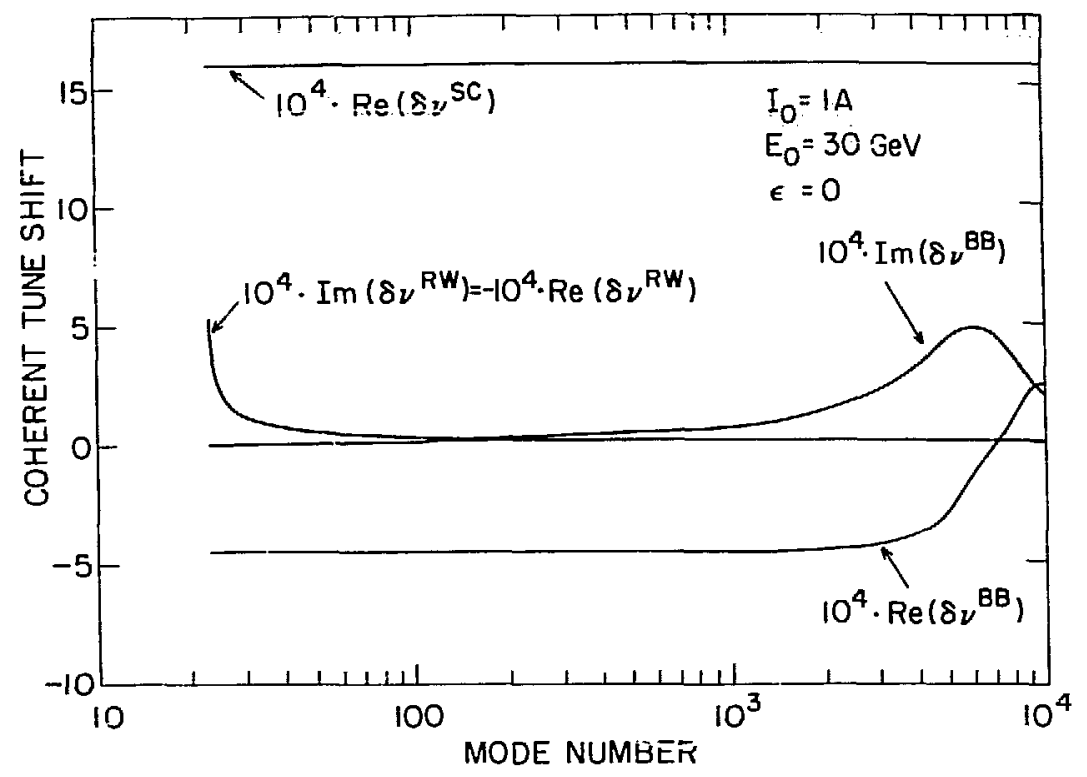

Figure la. Real and jmaginary parts of the coherent tune shifts of an on-axis beam.

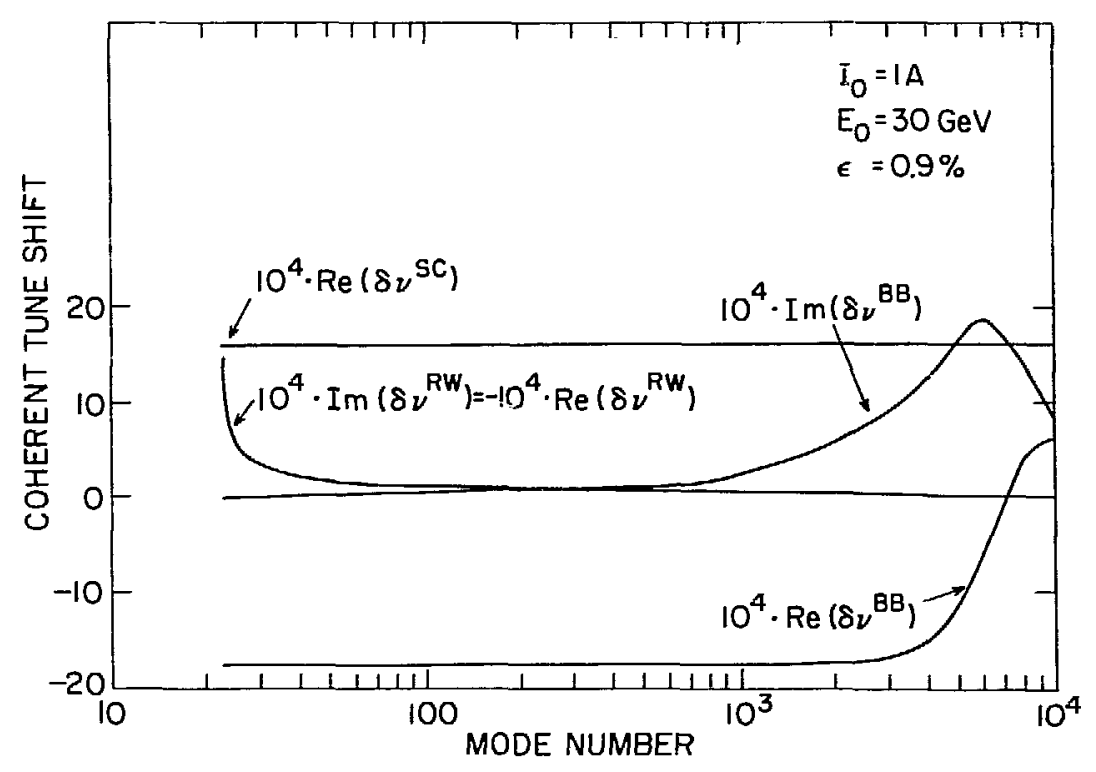

Figure lb. Coherent tune shifts for a beam which is $2 \mathrm{~cm}$ off-axis, corresponding to a momentum deviation of $0.9 \%$. 
we have, for $\varepsilon_{\mathrm{n}}$ real, and using Eq. (49),

$$
\begin{aligned}
\int \mathrm{d} \varepsilon \frac{g_{o}(\varepsilon)}{\varepsilon-\varepsilon_{n}}= & \frac{3}{4 \varepsilon_{M}}\left\{\left[1-\left(\frac{\varepsilon_{n}}{\varepsilon_{M}}\right)^{2}\right] \text { ln }\left|\frac{\varepsilon_{M}-\varepsilon_{n}}{\varepsilon_{M}+\varepsilon_{n}}\right|-2 \frac{\varepsilon_{n}}{\varepsilon_{M}}\right. \\
& \left.+i \pi\left[1-\left(\frac{\varepsilon_{n}}{\varepsilon_{M}}\right)^{2}\right] \theta\left(\varepsilon_{M}-\left|\varepsilon_{n}\right|\right)\right\}
\end{aligned}
$$

where $\theta$ is the step function. From this and $\mathrm{Eq} .(50)$ we have

$$
\begin{gathered}
\subseteq=\frac{3}{4 a_{2} \varepsilon_{M}}\left\{\left(1-x_{1}^{2}\right) \ln \left|\frac{1-x_{1}}{1+x_{1}}\right|-2 x_{1}\right\}, \\
P=\frac{3 \pi}{4 a_{2} \varepsilon_{M}}\left\{1-x_{1}^{2}\right\} \theta\left(1-\left|x_{1}\right|\right)
\end{gathered}
$$

with

$$
\mathrm{x}_{1}=-\frac{\mathrm{a}_{1}}{\mathrm{a}_{2}}=-\frac{\nu-v_{0}}{\mathrm{a}_{2} \varepsilon_{1}} .
$$

The $I_{o Q}$ and $I_{o p}$ currents are obtained from Eqs. (52), (53), (72), and (73) as

$$
\begin{gathered}
I_{O Q}=-\frac{2 S}{3} \frac{A}{A^{2}+B^{2}-\left(1-x_{1}^{2}\right) ! n\left|\frac{1-T^{1}}{I F X_{1}}\right|-2 x_{1}} \\
I_{O P}=\frac{2 S}{3 \pi} \frac{B}{A^{2}+B^{2}} \frac{1}{\left(1-x_{1}^{2}\right) \theta\left(1-\left|x_{1}\right|\right)}
\end{gathered}
$$

where

$$
S=-2 a_{2} \varepsilon_{M}>0
$$

is the energy spread.

To calculate explicitly the current, let us consider a case such that

$$
\left(\delta v_{p} / I_{o}\right)=6.3 \times 10^{-4}+i 5.2 \times 10^{-4}
$$

(this describes ISABELLE for $\mathrm{p}=-23$ and $\mathrm{E}_{\mathrm{o}}=30 \mathrm{GeV}$ and $v_{\mathrm{o}}=22.6$, $a_{2}=-2$ ). 
This value is obtained from Figure 1 , summing the various terms. We have now from Eqs. (75), (76), and (77)

$$
\begin{gathered}
I_{O Q}=-6.3 \times 10^{2} \mathrm{~s} /\left\{\left(1-x_{1}^{2}\right) \ln \left|\frac{1-x_{1}}{1+x_{1}}\right|-2 x_{1}\right\}, \\
I_{O P}=1.65 \times 10^{2} \mathrm{~s} /\left(1-x_{1}^{2}\right) \theta\left(1-\left|x_{1}\right|\right) .
\end{gathered}
$$

In Figure 2 we have plotted the currents, $I_{O Q}, I_{O P}$ versus $x_{1}$. We see that there is a solution for $X_{1} \cong 0.64$ corresponding to a threshold current, $I_{t h}$,

$$
\frac{I_{t h}}{S} \simeq 280
$$

For a stable current of $8 \mathrm{~A}$ this implies a spread $\mathrm{S} \cong 3 \times 10^{-2}$ and a tune shift $v-v_{0}=\mathrm{SX}_{1} / 2=9 \times 10^{-3}$.

We consider now the case $a_{3} \neq 0, a_{n}=0$ for $n>3$. Using Eqs. (56), (57), and (59) we write the dispercion relation as

$$
1=\delta \nu_{\mathrm{p}} \frac{1}{\mathrm{a}_{3}\left(\varepsilon_{1}-\varepsilon_{2}\right)} \int \mathrm{d} \varepsilon g_{0}(\varepsilon)\left\{\frac{1}{\varepsilon-\varepsilon_{1}}-\frac{1}{\varepsilon-\varepsilon_{2}}\right\}
$$

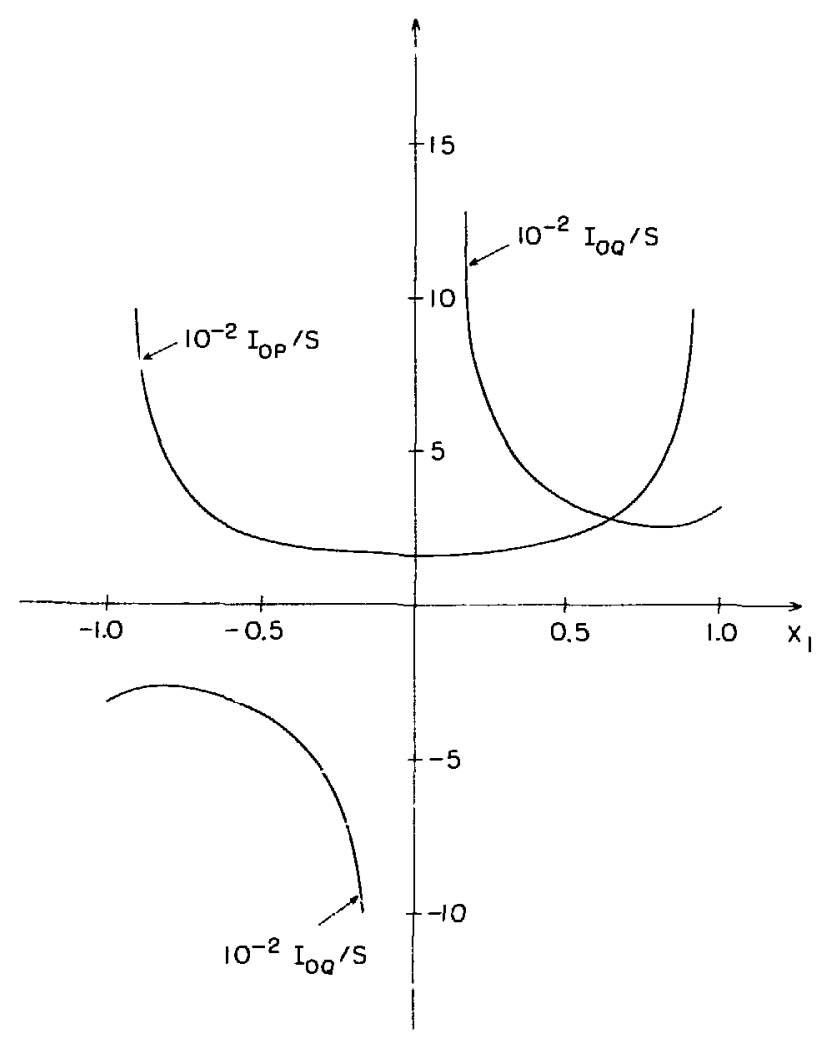

Figure 2. $\mathrm{I}_{\mathrm{oP}} / \mathrm{S}$ and $\mathrm{I}_{\mathrm{OQ}} / \mathrm{S}$ as functions of $x_{1}$. 
To evaluate Eq. ( 81 ) we have to consider two cases:

(a)

$$
a_{2}^{2}-4 a_{1} a_{3}<0, \quad a_{1} \text { real }
$$

in which the dispersion integral has only a real part, i.e., $Q \neq 0, P=0$; there is no stable solution but for the case $\operatorname{Im} \delta v_{\mathrm{p}}=0$.

$$
a_{2}^{2}-4 a_{1} a_{3}>0, \quad a_{1} \text { real }
$$

in which both $Q$ and $P$ are non zero. In case (b), taking the limit $a_{1} \rightarrow 0^{+}$, the dispersion relation becomes

$$
1=\frac{\delta v_{p}}{a_{3}\left(\varepsilon_{1}-\varepsilon_{2}\right)}\left\{P \int d \varepsilon g_{0}(\varepsilon)\left[\frac{1}{\varepsilon-\varepsilon_{1}}-\frac{1}{\varepsilon-\varepsilon_{2}}\right]-i \pi \varepsilon_{0}\left(\varepsilon_{1}\right)-i \pi g_{0}\left(\varepsilon_{2}\right)\right\}
$$

which gives

$$
\begin{aligned}
Q=\frac{3}{4 \varepsilon_{M} a_{3}\left(\varepsilon_{1}-\varepsilon_{2}\right)}\left\{\left[1-\left(\frac{\varepsilon_{1}}{\varepsilon_{M}}\right)^{2}\right] \text { ln }\left|\frac{1-\varepsilon_{1} / \varepsilon_{M}}{1+\varepsilon_{1} / \varepsilon_{M}}\right|-2 \frac{\varepsilon_{1}}{\varepsilon_{M}}\right. \\
\left.-\left[1-\left(\frac{\varepsilon_{2}}{\varepsilon_{M}}\right)^{2}\right] \text { \&n }\left|\frac{1-\varepsilon_{2} / \varepsilon_{M}}{1+\varepsilon_{2} / \varepsilon_{M}}\right|+2 \frac{\varepsilon_{2}}{\varepsilon_{M}}\right\}
\end{aligned}
$$

and

$$
\begin{aligned}
\left.P=-\frac{3 \pi}{4 \varepsilon_{M} a^{a}\left(\varepsilon_{1}\right.}-\varepsilon_{2}\right) & \left\{\left[1-\left(\frac{\varepsilon_{l}}{\varepsilon_{M}}\right)^{2}\right] \theta\left(\varepsilon_{M}-\left|\varepsilon_{1}\right|\right)\right. \\
& \left.+\left[1-\left(\frac{\varepsilon_{2}}{\varepsilon_{M}}\right)\right] \theta\left(\varepsilon_{M}-\left|\varepsilon_{2}\right|\right)\right\} .
\end{aligned}
$$

Note that for $a_{3} \rightarrow 0, a_{2}>0,\left|\varepsilon_{2}\right| \rightarrow \infty, \varepsilon_{1} \rightarrow-a_{1} /\left|a_{2}\right|$, and we have

$$
\begin{gathered}
Q=\frac{3}{\left.4 \varepsilon_{M}\right|_{2} \dagger}\left\{\left[1-\left(\frac{\varepsilon_{1}}{\varepsilon_{M}}\right)^{2}\right] \ln \left|\frac{1-\varepsilon_{1} / \varepsilon_{M}}{\Gamma+\varepsilon_{2} / \varepsilon_{M}}\right|-2 \frac{\varepsilon_{1}}{\varepsilon_{M}}+0\left(\frac{\varepsilon_{M}}{\varepsilon_{2}}\right)\right\} \\
P=-\frac{3 \pi}{4 \varepsilon_{M} a_{2} T}\left[1-\left(\frac{\varepsilon_{1}}{\varepsilon_{M}}\right)^{2}\right] \theta\left(1-\left|\frac{\varepsilon_{1}}{\varepsilon_{M}}\right|\right)
\end{gathered}
$$

which agrees with Eqs. (72) and (73) also taking into account the sign of $a_{2}$.

For $a_{2}<0$ we have to exchange $\varepsilon_{1}$ with $\varepsilon_{2}$, and, for $a_{3} \rightarrow 0, \varepsilon_{2} \rightarrow a_{1} /\left|a_{2}\right|$, $\varepsilon_{1} \rightarrow \infty$.

To evaluate $Q$ and $P$ let us introduce the quantities

$$
\mathrm{u}_{1}=\varepsilon_{1} / \varepsilon_{\mathrm{M}}, \quad \mathrm{u}_{2}=\varepsilon_{2} / \varepsilon_{\mathrm{M}}
$$




$$
\begin{aligned}
& u_{1}+u_{2}=\frac{a_{2}}{\varepsilon_{M^{a} 3}} \equiv-\frac{1}{\Delta}, \\
& \mathrm{S}=-2 \mathrm{a}_{2}{ }^{-} \mathrm{M}, \\
& Q=-\frac{2}{S} \text {, } \\
& P=-\frac{2}{S} \text {, } \\
& R=\frac{3}{4 \Delta\left(u_{1}-u_{2}\right)}\left\{\left[1-u_{1}^{2}\right] \text { ln }\left|\frac{1-u_{1}}{1+u_{1}}\right|-2 u\right. \\
& \left.-\left[1-u_{2}^{2}\right] \ln \left|\frac{1-u_{2}}{1+u_{2}}\right|+2 u_{2}\right\}, \\
& I=-\frac{3 \pi}{4 \Delta\left(u_{1}-u_{2}\right)}\left\{\left[1-u_{1}^{2}\right] \theta\left(1-\left|u_{1}\right|\right)\right. \\
& \left.+\left[1-u_{2}^{2}\right] \theta\left(1-\left|u_{2}\right|\right)\right\} \text {. }
\end{aligned}
$$

Using $R$ and $I$ we can rewrite the dispersion relation as

$$
\begin{aligned}
& \frac{2 I_{O Q}}{S}=-\frac{1}{R} \frac{A}{A^{2}+B^{2}}, \\
& \frac{2 I_{O Q}}{S}=\frac{1}{I} \frac{B}{A^{2}+B^{2}} .
\end{aligned}
$$

Let us now apply these formulae to the case of ISABELLE. To evaluate $S$ and $\Delta$ we assume

$$
a_{2}=-2
$$

giving a total frequency spread (sextupole), for the case $\Delta E / E=\varepsilon_{M}=0.5 \%$, of $\mathrm{S}=2 \%$. The term $\mathrm{a}_{3}$ is given by

$$
a_{3}=\frac{v_{o}}{2} \bar{b}_{3,1} x_{p}^{2}
$$

with

$$
\bar{b}_{3,1}=\frac{b_{3,1}}{v_{0}^{2}}-2 \frac{\Delta v_{s c}}{v_{0}} \frac{s_{3,1}}{s_{1,1}} .
$$

We consider only the effect of space charge $\left(b_{3,1}=0\right)=$ giving

$$
a_{3}=-\Delta v_{s c} \frac{s_{3,1} x_{p}^{2}}{s_{1,1}}
$$


The incoherent space-charge tune shift for ISABELLE can be written as

$$
\delta v_{\mathrm{sc}}=\Delta v_{\mathrm{sc}}\left(1+\frac{\mathrm{s}_{3,1}}{\mathrm{~S}_{1,1}} \mathrm{x}_{\mathrm{p}}^{2} \varepsilon^{2}\right)
$$

with

$$
\Delta v_{\mathrm{Sc}}=-1.6 \times 10^{-3} \mathrm{I}_{\mathrm{o}}
$$

and

$$
\Delta v_{\text {sc }} \frac{S_{3,1}}{S_{1,1}} X_{p}^{2}=\frac{3 \times 10^{-3}}{\varepsilon_{M}^{2}}
$$

Note that the first term is negative (direct space charge larger than image effects, small neutralization) and is proportional to the current. The second term comes from the regular lattice cells, where $x_{p} \neq 0$ and the beam size is proportional to $\varepsilon_{M}$ and to the current. As a result this term does not depend on $I_{0}$.

With this assumptions we have

$$
\Delta=\frac{a_{3} \varepsilon_{M}^{2}}{a_{2} \varepsilon_{M}}=0.3 .
$$

For the coherent tune shift we again assume

$$
\delta v=6.3 \times 10^{-4}+15.2 \times 10^{-4}
$$

$\therefore$ orresponding to $\mathrm{p}=-23, \mathrm{E}=30 \mathrm{GeV}$

Using this impedance we have

$$
\begin{aligned}
& \frac{2 I_{O Q}}{S}=-\frac{944}{R}, \\
& \frac{2 I_{O P}}{S}=\frac{779}{I} .
\end{aligned}
$$

A solution with $I_{o Q}=I_{o P}$ can be obtained for

$$
\begin{gathered}
R<0, \quad I>0, \\
\quad \frac{I}{R}=-0.825 .
\end{gathered}
$$

The two functions $R$ and $I$ versus $u_{2}$ are plotted in Figures 3, 4, and 5 . Using these we can evaluate the threshold current versus $\Delta$, given in Figure 6 . Note that, for $A>0, I_{0}$ increases for $\Delta>0$ and decreases sharply for $\Delta<0$. The opposite would be true for $A<0$. In the ISABELLE case $A>0$ and $\Delta=0.3$, so that the effect of the space charge is to increase the threshold by about $20 \%$. 


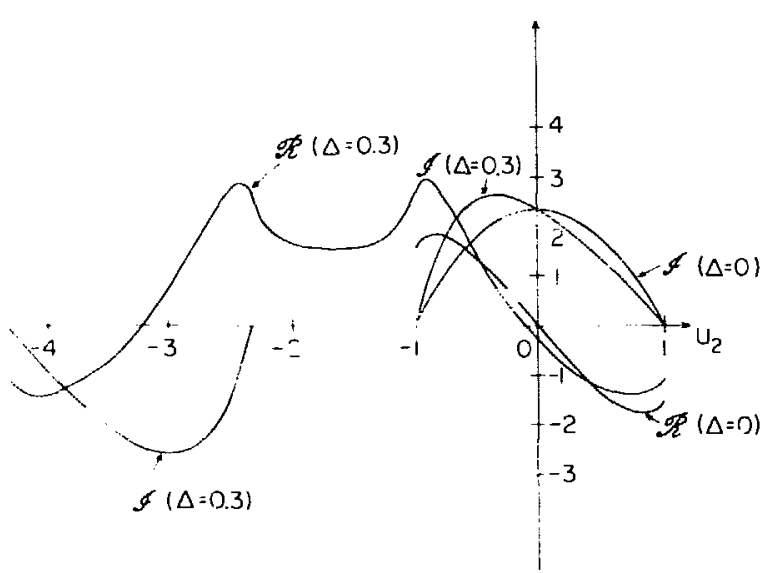

Figure 3. $I$ and $R$ functions versus $u_{2}$ for $\therefore=0$ and $\Delta=0.3$.

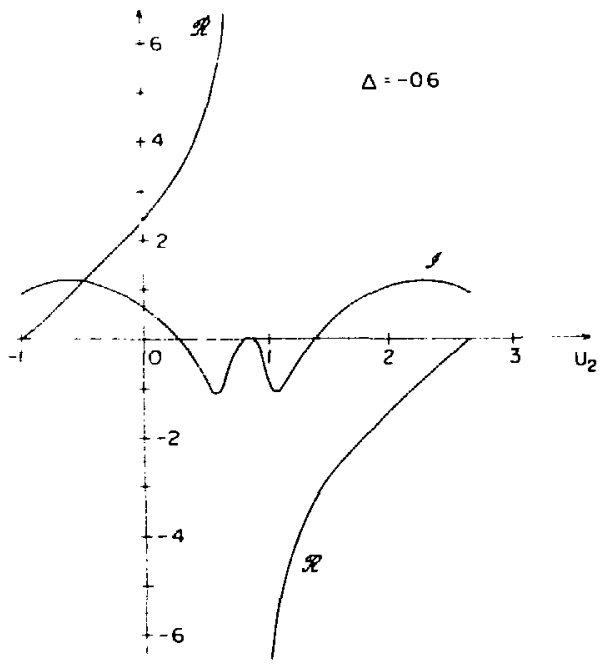

Figure 5. $T$ and $R$ functions versus $\mathrm{u}_{2}$ fur $\therefore=-0.6$.

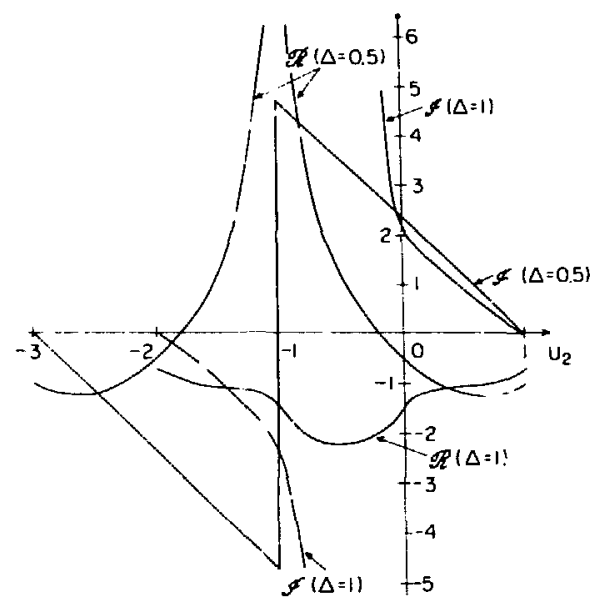

Figure $4 . \quad I$ and $R$ Functions versus $\mathrm{u}_{2}$ for $\dot{\mathrm{S}}=0.5$ and $\mathrm{L}=1$.

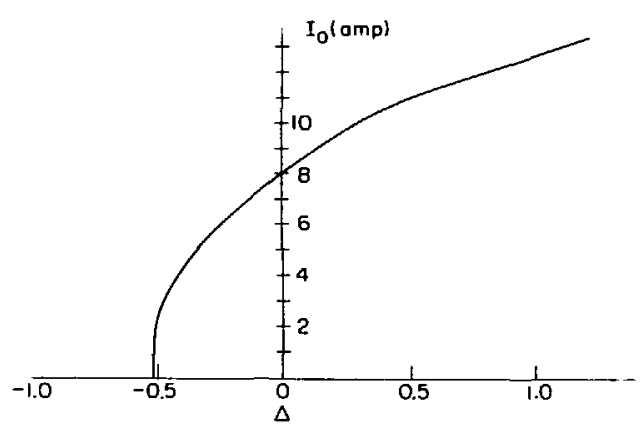

Figure 6. Threshold current as a function of $i$. 
Note also that for $A>0$ the solution of the dispersion relation gives $a_{1}=v-v_{0}>0$ and the coherent tune falls in the part of the frequency distribution corresponding to $\varepsilon>0$. The local chromaticity at the coherent tune is increased if $\Delta>0$, corresponding to an increase in current, or decreased if $\Delta<0$. We will see in Part II that this relationship between the threshold current and the value of the chromaticity at the coherent tune is valid for all the frequency distributions that we consider.

PART II

CONDITIONS FOR TRANSVERSE STABILITY IN ISABELLE

\section{Numerical Integration of the Dispersion Relation}

An analytical solution of the dispersion relation (39) can be obtained only if we limit the order of the polynominal $\sum_{n} \varepsilon^{n}$ to two or three and use a simple expression for the energy distribution function $g_{0}(\varepsilon)$. These limitations have been removed by using a numerical technique to solve (39).

A computer program has been written which, for a given set of multipoles, $b_{n, 1}$, and space-charge coefficients, $S_{n, l}$, finds the zeros of the polyneminal in'Eq. (59) and solves the dispersion rélation by the method described in Part I, ior any arbitrary moneatum distribution functions $g_{0}(\varepsilon)$. 'The principal part of the integral in $\mathrm{Eq}$. (61) has a singularity; in the numerical integration this singularity has been removed by avoiding a small interval. $\varepsilon_{\mathrm{n}} \pm \Delta \varepsilon$ in the path of integration.

The goal of the study is to determine the stability conditions of the ISABELLE beams, and the magnetic corrector requirements for the control of the working line. We have considered the following beam dynamics situations, corresponding to five different stages of the storage and acceleration cycle:

a) partially stacked beam close to the chamber wall,

b) fully stacked beam off axis,

c) fully stacked debunched beam at the center of the chamber,

d) bunched beam at the early stage of acceleration, and

e) electron neutralization effect at high energy.

The conditions for the instability are not too different in the horizontal and vertical planes. As mentioned in Appendix $A$, both the linear spacecharge tune shift and the spread are smaller in the horizontal plane. In the most critical case of an off-axis beam, a smaller linear coherent tunc shift reduces the beneficial cancellation with the wali induced tune shift, but, on the other hand, the negative effect of the curvature of the working line is considerably rediced. Because of the circular geometry, we may assume that the impedance presented to the beam by the environment is approximately the same in the horizontal and vertical planes.

In this report we will consider only the vertical instability, and will assume that the general conclusions apply to both planes. Given the degree of uncertainty in the basic assumptions, such as the transverse space-charge distribution and the impedance model, we feel that this inoroach is justified. 
For the sake of understanding the physics of the instability, and in order to link with the theoretical example discussed in Part I, we first consider case $(\dot{)})$, which is the most straightforward.

2. Fully Stacked Debunched Beam at the Center of the Chamber

The analytical solution for this case has been discussed in Part I. The model included a sextupole field and a space-charge term consisting of a linear and octupolar terms. For the lowest mode $p=-23$, both the real and imaginary part of the coherent tune shift are positive; the threshold ccndition was obtained with a sextupolar tune spread of 0.03 , or a chromaticity of 3 , for a current of $8 \mathrm{~A}$ with parabolic momentum distribution. It was also found that the octupolar space-charge term, because of its positive sign, actually increases the beam current threshold by $\sim 20 \%$. The simple physical explanation for this improvement is inat the octupolar space-charge term increases the slope of the local chromaticity at the positive edge of the momentum stack, where the coherent tune ${ }^{*}$ falls. If the real part of the coherent tune had negative sign, then the octupolar space--charge term would decrease the local chromaticity where it matters most, namely at the negative edge of the momentum stack, and, as shown in the theoretical model of Part $I_{2}$ a drastic decrease in current threshold would result.

Table I shows the beam current threshold for the conditions where other multipoles, besides sextupoles, are used to provide the tune spread for Landau damping. For simplicity we indicate the multipoles $b_{n, l}$, as defined in Eq. (7), as $b_{n}$, omitting the second index. The strength of each multipole is chosen for a beam current threshold comparable with the sextupole-only case, and is of the order of the one required for ISABELLE at the injection energy of $30 \mathrm{GeV}(8 \mathrm{~A})$. The momentum distribution is given by a flat region in the momentum range $-0.0045 \leqslant \varepsilon \leqslant 0.0045$; a biquadratic distribution function (see Appendix B) was assumed for the tails which, given the total momentum spread of \pm 0.005 , cover $10 \%$ of this spread. The space-charge term as described in Appendix $A$ is assumed in this calculation. The coherent tune shift has been calculated according to $\mathrm{Eq}$. (62) and Figure 1.

The third column of Table I indicates the clear advantage of providing the tune spread for Landau damping with high-order multipoles, since, for approximately the same beam current threshold, the tune spread in the beam decreases. The reason for this is made apparent by the numbers in the fifth column, which refer to the momentum deviation corresponding to the coherent tune. These numbers show that the coherent tune falls in all cases towards the end of the flat region, regardless of the multipole used to provide Landau damping, and that the chromaticity at this location is also approximately constant, for the same current. The high-order multipoles are able to provide the same local chromaticity (at the coherent tune location) with a smaller overall tune spread. The results also indicate that the value of this local

* In what follows we call "coherent tune" the quantity $a_{1}=v-v_{0}$. 
TABLE I

Beam Current Threshold of a Coasting Beam at the Center of the Vacuum Chamber for Various Mode Numbers and Magnet1c Multipoles

\begin{tabular}{|c|c|c|c|c|c|c|}
\hline $\begin{array}{c}\text { Mode } \\
\text { number }\end{array}$ & $\begin{array}{l}\text { Type of } \\
\text { correcting } \\
\text { multipoles } \\
\end{array}$ & $\begin{array}{c}\text { Total tune } \\
\text { spread in } \\
\text { beam (including } \\
\text { space charge octupole) }\end{array}$ & $\begin{array}{c}\text { Coherent } \\
\text { tune, } \\
\check{c}_{1}=v-v_{0}\end{array}$ & $\begin{array}{c}\text { Coherent tune } \\
\text { location in } \\
\text { momentum range } \\
\left(\varepsilon^{*}\right)\end{array}$ & $\begin{array}{l}\text { Chromaticity } \\
\text { at coherint } \\
\text { tune location }\end{array}$ & $\begin{array}{l}\text { Beam } \\
\text { current } \\
\text { threshold } \\
\text { (A) }\end{array}$ \\
\hline-23 & sextupole & 0.030 & 0.014 & 0.0040 & 3.9 & 11.3 \\
\hline$-\quad 23$ & octupole & 0.018 & $\cdots$ & 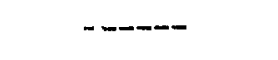 & -- & Unstable \\
\hline$-\quad 23$ & decapole & 0.019 & 0.006 & 0.0040 & 3.2 & 12.6 \\
\hline-23 & 14-pole & 0.016 & 0.004 & 0.0040 & 3.5 & 11.2 \\
\hline-23 & 18-pole & 0.011 & 0.007 & 0.0045 & 4.9 & 18.5 \\
\hline-50 & sextupole & 0.030 & 0.017 & 0.0050 & 3.8 & 7.4 \\
\hline-50 & decapole & 0.019 & 0.013 & 0.0050 & 6.5 & 12.5 \\
\hline-50 & 14-pole & 0.016 & 0.012 & 0.0050 & 8.3 & 15.8 \\
\hline-7000 & sextupole & 0.030 & 0.081 & $---\cdots$ & $-\cdots$ & 18.9 \\
\hline
\end{tabular}

* Relative momentum deviation $\varepsilon$ of a particle oscillating at the coherent tune. 
chromaticity is predominant in deciding the stability conditions, "nearly" regardless of the chromaticicy dependence with momentum at other points in the stack. An exception to this statement is represented by the odd multipoles (octupole in the table): for these multipoles, the computer results indicate that a non-monotonic chromaticity across the stack causes an unstable situation, which becomes increasingly worse as the order of the odd multipoles is increased.

The mode numbers in Table I are selected as representative of signifisant points in the impedance curves of Figure 1. In particular, the $p=-7000$ number curresponds to the cutoff limit. Table I shows that for large mode numbers the damping introduced by the revolution frequency spread increases the threshold current. In fact, the change in oscillation frequency due to the change in revolution frequency is dominant over that due to the change in turie. The effect of the momentum distribution function, denoted as $g_{0}(\varepsilon)$ in Part I on the beam stability is shown in Table II. As in the model described earlier, a coasting beam at $30 \mathrm{GeV}$ is assumed, having a half momentum spread of $\varepsilon_{\mathrm{m}}=0.005$. Two cases of a sextupole or decapole field providing rine tune spread have been considered.

Table II shows the expected reduction in current threshold for a parabolic momentum distribution with respect to an elliptical one. A parabolic momentum distribution has a sharper falloff. This, qualitatively speaking, pushes the coherent tune towards the center of the stack; in turn, a smaller coherent tune means a smaller threshold current. This effect is even more dramatic $i^{-}$the decapole case, since, as the coherent tune moves towards tha center of the stack, the chromaticity at this location drops, further reducing the current threshold. A flat distribution with tails is more stable than an elliptic or parabolic distribution. It is perhaps surprising that, in the range of values considered, longer tails reduce the threshold. However, it should be noted that the coherent tune falls at the edge of the flat region, and a longer tail involves a smaller coherent tune. Other computations not reported here show that, if the coherent tune falls in the tail of the momentum distributions, longer tails have a stronger stabilizing effect.

3. Bunched Beam at the Center of the Chamber

This case concerns the beam soon after captura by the radiofrequency system at harmonic number 3 . This situation corresponds to the maximum value of the ratio peak current/energy, having taken into account the detailed description of the acceleration process. 5 At $30 \mathrm{GeV}$, and before acceleration starts, the peak current is about $12 \mathrm{~A}$ in each bunch. We assume that the theory for coasting beams can be applied to a bunched beam for a wavelength of the perturbing field which is small compared with the bunch length. The mode number -23 , lowest mode for the instability, should satisfy the above condition.

5 E. Ezura, private communication. 
TABLE II

Effect of various distribution functions $g_{0}(\varepsilon)$ on the beam stability (Mode Number $=-23$ )

\begin{tabular}{|c|c|c|c|c|c|}
\hline $\begin{array}{l}\text { Distribution } \\
\text { Function } \\
\end{array}$ & $\begin{array}{c}\text { Coherent } \\
\text { Tune } \\
\end{array}$ & $\begin{array}{l}\text { Coherent Tune } \\
\text { Location in } \\
\text { Momentum } \\
\text { Range (E) }\end{array}$ & $\begin{array}{l}\text { Chromaticity } \\
\text { at Coherent } \\
\text { Tune Location }\end{array}$ & Multipole & $\begin{array}{c}\text { Beam } \\
\text { Currant } \\
\text { Threshold }\end{array}$ \\
\hline $\begin{array}{l}\text { Flat with } 5 \% \\
\text { biquadratic tail }\end{array}$ & 0.014 & 0.0040 & 3.9 & sextupole & 11.7 \\
\hline $\begin{array}{l}\text { Flat with } 10 \% \\
\text { biquadratic tail }\end{array}$ & 0.014 & 0.0040 & 3.9 & sextupole & 11.3 \\
\hline $\begin{array}{l}\text { Flat with } 20 \% \\
\text { biquadratic tail }\end{array}$ & 0.013 & 0.0038 & 3.8 & sextupole & 10.7 \\
\hline E1liptic & 0.009 & 0.0025 & 3.7 & sextupole & 12.4 \\
\hline Parabolic & 0.008 & 0.0025 & 3.5 & sextupole & 9.9 \\
\hline $\begin{array}{l}\text { Flat with } 5 \% \\
\text { biquadratic tail }\end{array}$ & 0.007 & 0.0040 & 4.1 & decapole & 13.8 \\
\hline $\begin{array}{l}\text { Flat with } 10 \% \\
\text { biquadratic tall }\end{array}$ & 0.006 & 0.0039 & 3.8 & decapole & 12.6 \\
\hline $\begin{array}{l}\text { Flat with } 20 \% \\
\text { biquadratic tail }\end{array}$ & 0.005 & 0.0036 & 3.2 & decapole & 10.9 \\
\hline Elliptic & 0.002 & 0.0028 & 2.0 & decapole & 6.7 \\
\hline Parabolic & 0.001 & 0.0022 & 1.2 & decapole & 4.1 \\
\hline
\end{tabular}


For this mode, we find that a sextupole providing a chromaticity of 2 , and a corresponding tune spread of \pm 0.017 over the assumed momentum spread of $2 \varepsilon_{M}=1.74 \times 10^{-2}$, satisfies the stability conditions for $12-\mathrm{A}$ peak current. A parabolic charge distribution was assumed. For such a distribution, the coherent tune of the lowest mode $p=-23$ falls halfway between the center and the end of the positive side of the momentum width, i.e., at $\varepsilon=4.4 \times 10^{-3}$. This location of the coherent tune shift has some important effects: the octupolar space-charge term, which, as shown in the previous paragraph, helped to raise the threshold of the instability in the coasting beam case, is now less effective. More important, the benefit of using higher-order multipoles is lost. As an example, let us take a decapole field which provides the same local chromaticity as a sextupole field at the coherent tune location. In this case, the beam current threshold is the same as in the sextupole case (12 A), but the tune spread in the beam increases to $\sim 0.03$.

\section{Partially Stacked Beam}

Consider a partially stacked beam, offset with respect to the vacuum chamber axis. We consider the case of a beam having a momentum width $\varepsilon_{\mathrm{m}}=0.001$, centered at $\varepsilon_{\mathrm{d}}=0.009$. A rectangular momentum distribution with $20 \%$ biquadratic tails is assumed (Figure 7 ). The required threshold current in this case is $1.6 \mathrm{~A}$.

The offset in the horizontal position of the beam in the vacuum chamber changes the resistive and broad-band impedance terms, as discussed in Appendix $C$ and Figure 1. The main effect is a reverse in the sign of the real term of the impedance, which causes a shift of the coherent tune to the negative side of the momentum stack compared with the centered beam case. The results of the study are summarized in Figures 8 to 10 . Figure 8 shows some examples of tune versus momentum curves which give stability for a $1.6 \mathrm{~A}$ beam for the mode number $p=-23$. At the coherent tune location a chromaticity of 13.5 is the minimum required for stability. Because of the location of the

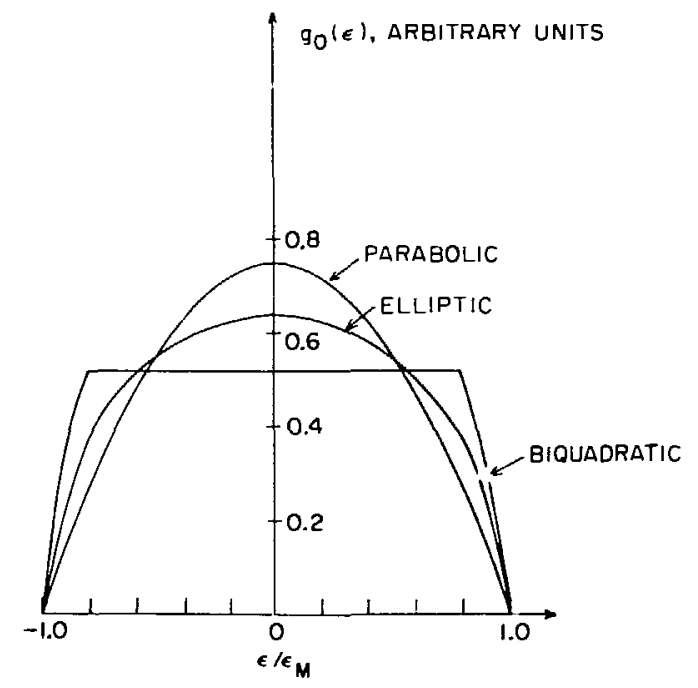

Figure 7. Various types of momentum distribution functions. 


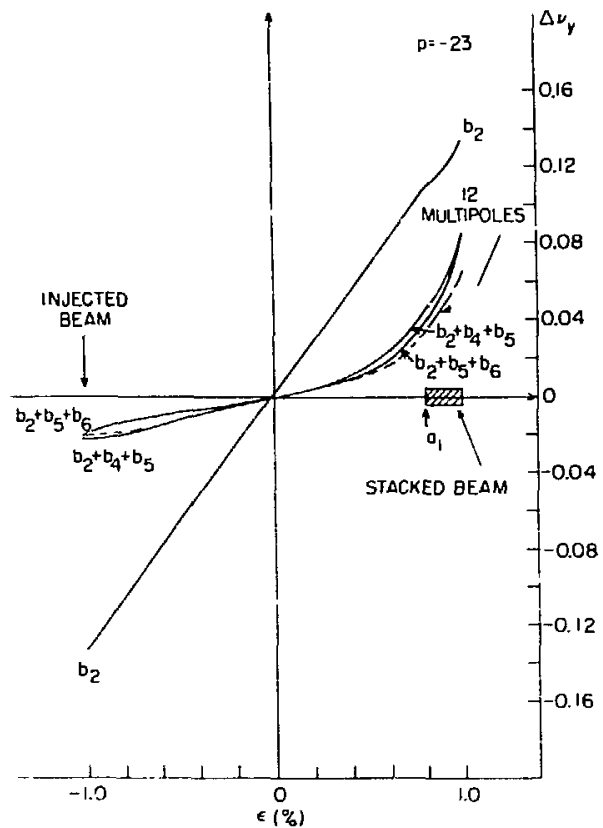

Figure 8 . Tune shift across the momentum aperture required for the stability of a 1.6-A stacked beam, having an average momentum deviation of $0.9 \%$. Chromaticity of about 2 is assumed to be required for the stability of the bunched injected beam. Mode number $=-23$.

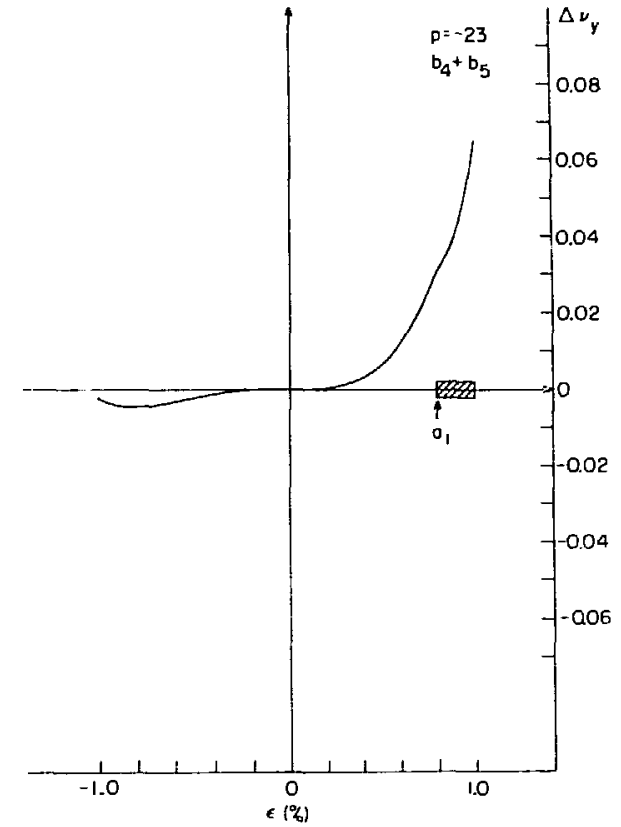

Figure 9. Tune shift across the momentum aperture required for the stability of a 1.6-A stacked beam, having an average momentum deviation of $0.9 \%$. A nearly zero chromaticity is supposed to be acceptable for the bunched injected beam. Mode number $=-23$.
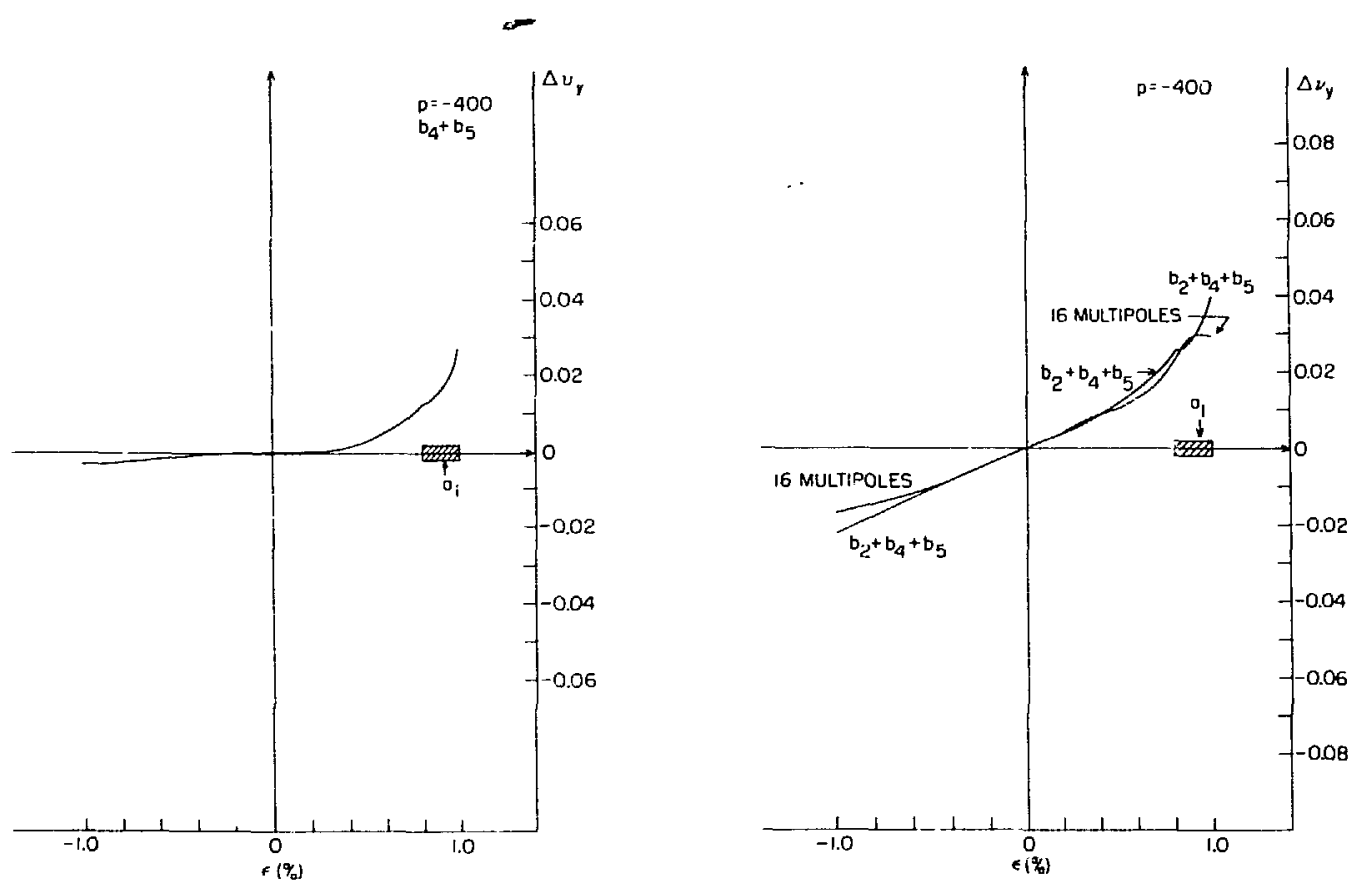

Figure 10. Tune shif: xcross the momentum aperture required for the stability of a 1.6-A stacked beam, having in average momentum deviation of $0.9 \%$. Mode number $=-23$. 
coherent tune, denotfd as $a_{1}$ in Figure 8 , the space-charge octupole lowers the threstold of the beam current, unless its negative chromaticity on the negative side of the momentum stack is compensated by the magnetic multipoles. However, this correction in turn increases the tune spread at the positive edge of the momentum stack, and the curves show that there is little to be gained by increasing the multipole corrector above dodecapole $\left(b_{5}\right)$. The "12 multipole" case (up to and including $b_{12}$ ) is an attempt to force a reduction in chromaticity, and thus in tune spread, at the positive edge of the stack, corresponding to $\varepsilon=0.01$. As can be seen in Figure 8 , this reduces the tune spread by $\sim 0.02$. In all cases, a pair of consecutive multipoles $\left(b_{4}+b_{5}, b_{5}\right.$ $+b_{6}$, etc.) is required in order for the chromaticities to substract at $\varepsilon=$ -0.01. This is the location of the injected bunch from the AGS. In the stacking process, the bunch has to traverse the whole tune range from $\varepsilon=$ -0.01 to $\varepsilon=+0.008$ (in our example). Thus, it is important to keep the tune shift across the momentum aperture small. Iil Figure 8 we assume that a positive chromaticity of +2 is required for the injected beam, and this is provided by a sextupole term. In Figure 9 we have considered the case where the chromaticity can be reduced to $\sim 0$ in the bunched beam region. It is quite llear that, in both cases, a large tune spread will be required, of the order of 0.07 at best. To reduce this tune spread, it will probably be necessary to cure the instability at the lowest modes with a feedback system. If we assume a bandwidth of the feedback system of $\sim 30 \mathrm{MHz}$, then the lowest mode of concern is $p \approx-400$. The cancellation of the space-charge and broad-band impedance terms (see Appendix B) for this mode number results in small values of the real and imaginary parts of the coherent tune shift. The local chromaticity required for stability is only $\sim 1$; however, the space sharge field, which gives a chromaticity $\sim 6$ at the edge of the stack, plays a major role and must be compensated.

The beneficial effect of the revolution frequency spread is significant at this mode number. Figure 10 shows the tune spread requirement for stability for the following cases:

a) No sextupole required for the injected beam at $\varepsilon=-0.01$. Decapole and dodecapole magnets provide the tune spread (Figure 10, left). Total tune spread required (in the beam region, $\varepsilon= \pm 0.01$ ) $=0.03$.

b) As above, with an additional sextupole to provide a chromaticity of 2 for the injected beam (Figure 10, right). Total tune spread required (in the beam region) $=0.06$.

i) A reduction in chromaticity at the positive edge of the momentum stack can be tolerated without reducing the current threshold, since the coherent tune shift falls sufficiently far away from this edge. However, in order to achieve a resultant zero chromaticity at $\varepsilon=0.01$, and the required positive chromaticity of $\sim 1$ at the coherent tune shift location, a total of $16 \mathrm{multipoles}$ are required (Figure 10, right).

The result of the study is that, for the given impedance model, there is little to be gained by increasing the number of multipoles above dodecapole, 
unless one is able to introduce a very large number of multipoles (up to and including $b_{16}$ ). Even in this case, the tune spread reduction tould be only 0.01 compared with the case where only the first five multipoles (up to and including $b_{5}$ ) are used.

\section{Fu11y Stacked Beam, Off-Axis}

As the momentum stack bullds up, the center of charge of the beam moves away from the chamber wall, towards the center. For this case, we have

$$
\begin{aligned}
& \varepsilon_{\mathrm{d}}=0.005, \\
& \varepsilon_{\mathrm{M}}=0.005, \\
& \text { Beam current threshold }>8 \mathrm{~A}, \\
& \left(\frac{\delta \nu_{\mathrm{p}}}{\mathrm{I}_{\mathrm{o}}}\right)_{\text {real }}=-6.7 \times 10^{-5} ;\left(\frac{\delta \nu_{\mathrm{P}}}{\mathrm{T}_{\mathrm{o}}}\right)_{\mathrm{imag}}=7.7 \times 10^{-4} .
\end{aligned}
$$

A threshold current of $9.6 \mathrm{~A}$ is obtained, for the mode $\mathrm{p}=-23$, with a constant chromaticity of 3 across the stack. This gives a tune spread of 0.03. An even smaller tune spread will be required for mode numbers above the pass-bend of the feedback system $(p<-400)$. Thus, the stability condition eases as the stack is being built up.

\section{Partially Neutralized High Energy Beam}

Let us consider a coasting proton beam at $400 \mathrm{GeV}$. A partial neutralization of the protons due to trapped electrons creates a field which distorts the working line and affects the transverse stability conditions. Moreover, the coherent tune shift associated with the space charge of the neutralized electrons must now be taken into account in the dispersion relation.

In Appendix A we calculate the coherent tune shift and the octupolar spread as a function of the fractional neutralization $f$. If we assume

$$
\mathrm{f}=2 \times 10^{-4} \text {, }
$$

then

$$
\delta v_{\mathrm{coh}}=-3.7 \times 10^{-3}
$$

and

$$
\delta v(\text { spread })=6 \times 10^{-4},
$$

the octupole being of negative sign (tune decreases for increasing momentum deviation).

The stability conditions are found with a sextupole field providing a vertical chromaticity $c_{v} \sim 15$. This rather strong requirement on the sixtupoles is due to the large value of the coherent tune shift. The variation of tune with momentum due to the octupolar space-charge term is 
small (its contribution to the chromaticity at the edge of the beam is only 3.2), and, moreover, has a favorable effect on the stablitity, since it increases the local chromaticity at the negative side of the momentum spread, where the coherent tune shift occurs. The tune spread in the beam is 0.012 , rather large for a $400-\mathrm{GeV}$ beam. Multipoles of order higher than sextupoles would not be effective, because of the small momentum spread (unless of course they are made unreasonably strong).

\section{Discussion on the Beam Dynamics Assumptions and Impedance Mode1}

The results presented so far are based on certain assumptions and on a model for the interaction of the beam with itself and the environment. In what follows we review these assumptions and make predictions based on a "worst case" situation.

Case a) Fu11y stacked beam at the center of the chamber The favorable situation arising from our impedance model is that a positive coherent tune, together with the space-charge occupole, in fact helps raise the instability threshold. If we assume that, at $30 \mathrm{GeV}$, the protons are partially neutralized, then the space-charg :induced coherent tune shift adds, in its real part, to the wall and the broad-band impedance contribution. For this condition the fractional neutralization must satisfy

$$
f \geq 1 / \gamma^{2} ;
$$

thus, at $30 \mathrm{GeV}, \eta \geqslant 1 \times 10^{-3}$ (an unlikely high neutralization!). Let is assume, for the sake of argument, $f=1 \times 10^{-3}$. Under this condition the beam is fully neutralized and, for the mode number $p=$ -23 ,

$$
\frac{\delta v_{p}}{I_{o}}=1.10 \times 10^{-3}+i 0.59 \times 10^{-3}
$$

The stability condition is satisfied, for a current of $8 \mathrm{~A}$, with a sextupole field providing a chromaticity of 5 and a total tune spread of 0.05 . A higher multipole would help reduce this spread since the coherent tune falls in this case at the negative edge of the stack. If the beam is not neutralized, but the real part of the coherent tune is still negative (as could happen if our model were incorrect), then a negative ehromaticity for negative momentum deviation would be desirable, since it would be increased by the negative shromaticity of the octupolar space charge term.

\section{Case b) Bunched beam}

The same considerations as for Case a) apply to the bunched beam, except that neutralization of the beam appears more unlikely. In any case, for the same conditions as discussed above, approximately the same increase in chromaticity is required. 
Case c) Partially stacked beam

For the lowest mode number, $\mathrm{p}=-23$, the impedance model we have used already presents a pessimistic view, since the coherent tune falls on the negative side of the momentum stack. For mode numbers above our assumed damper region $(p=-400)$, the beneficial effect of the revolution frequency spread is felt, in that the coherent tune shift is pushed towards the region of positive momentum and the tune spread requirement becomes less severe.

Case d) Neutralization of the beam at high energy

Our estimate of the fractional neutralization, $f=2 \times 10^{-4}$, is rather pessimistic, and the expectations are that this is an upper limit. Increasing the momentum spread reduces the octupolar term, but has a smaller effect on the coherent tune shift, to which the insertion regions, which are dispersion free, contribute by $\sim 50 \%$. Since the sign of the octupole term and the sign of the coherent tune shift are the same, we expect that the space-charge distortion of the working line will in fact always help raise the threshold of the instability.

8. Discussion of Space Charge Effects in the ISR and ISABELLE

It is well known that space-charge compensation is a very critical process in the ISR, so much so that the current threshold would be dramatically reduced without the use of pole face windings to straighten the working line.

In the ISR the direct space charge effect san be neglected, since it appears that $1 / \gamma^{2} \sim \mathrm{f}$ at $26 \mathrm{GeV} / \mathrm{s}$. Thus, the incoherent space-charge tune shifi in the ISR comes exclusively from the image charges, and the following empirical formulae, deduced from measurements, app $1 \mathrm{y}^{6}$ at $26 \mathrm{GeV} / \mathrm{c}$ :

$$
\begin{gathered}
\delta v_{\mathrm{y}}^{\mathrm{inc}}=-1.8 \times 10^{-3} \mathrm{I}_{\mathrm{o}}\left[1-\frac{0.2}{\varepsilon_{\mathrm{M}}^{2}}\left(\varepsilon-\varepsilon_{\mathrm{d}}\right)^{2}\right], \\
\delta v_{\mathrm{x}}^{\mathrm{inc}}=0.8 \delta v_{\mathrm{y}}^{\text {inc }} .
\end{gathered}
$$

In ISABELLE the indirect space charge effects are negligible compared with the direct space-charge effects (see Appendix A). The expression for the latter has been estimated to be

$$
\begin{gathered}
\delta v_{y}^{\text {inc }}=-1.6 \times 10^{-3} I_{o}+\frac{3 \times 10^{-3}}{\varepsilon_{M}^{2}}\left(\varepsilon-\varepsilon_{d}\right)^{2}, \\
\delta v_{x}^{\text {inc }} \sim-1.6 \times 10^{-3} I_{o} .
\end{gathered}
$$

6. G. Guignard, Selection of Formulae Concerning Proton Storage Rings, CERN, 77-10. 
For a $20 \%$ stacked beam, assuming

$$
I_{o}(I S R)=6 \mathrm{~A}
$$$$
\varepsilon_{M}(\text { ISR })=0.003
$$

$I_{O}($ ISA $)=1.6 \mathrm{~A}$
$\varepsilon_{M}($ ISA $)=0.001$

we find

Iinear tune shift:

ISR $=-0.011$

vertical

$=0.009$

horizontal

ISA $=-2.6 \times 10^{-3}$

vertical

$=-2.6 \times 10^{-3}$

horizontal

tune spread:

$$
\begin{aligned}
\text { ISR } & =2.2 \times 10^{-3} \\
& =-1.8 \times 10^{-3} \\
\text { ISA } & =3 \times 10^{-3} \\
& \simeq 0
\end{aligned}
$$

vertical

horizontal

vertical

\begin{tabular}{|c|c|c|c|}
\hline \multirow[t]{2}{*}{ linear tune shift: } & ISR & $\begin{array}{r}=-0.055 \\
=0.045\end{array}$ & $\begin{array}{l}\text { vertical } \\
\text { horizontal }\end{array}$ \\
\hline & ISA & $\begin{array}{l}=-0.013 \\
=-0.013\end{array}$ & $\begin{array}{l}\text { vertical } \\
\text { horizontal }\end{array}$ \\
\hline \multirow[t]{2}{*}{ tune spread: } & ISR & $\begin{array}{l}=0.011 \\
=-0.009\end{array}$ & $\begin{array}{l}\text { vertical } \\
\text { horizontal }\end{array}$ \\
\hline & ISA & $\begin{array}{l}=3 \times 10^{-3} \\
=0\end{array}$ & $\begin{array}{l}\text { vertical } \\
\text { horizontal }\end{array}$ \\
\hline
\end{tabular}

horizontal

For the full stack we find

The comparison indicates that the linear tune shift in ISABELLE is about a factor of 4 smaller than in the ISR, at all stages of the stacking process. This indicates perhaps that ISABELLE will be less sensitive to the crossing of nonlinear resonances during the stacking process. On the other hand, the curvature of the working line should be more pronounced in ISABELLE than in the ISR in the early stages of stacking, becoming progressively less important, since the spread remains the same but the width of the stack increases. It must be noted that, in the ISR, the horizontal and vertical space-charge octupoles have opposite sign, so that at least one of the two planes has an unfavorable curvature for a given impedance and needs to be corrected. In ISABELLE, only the vertical plane has a significant octupolar term, which will make it easier to compensate with our set of orthogonal multipolar correctors. 


\section{CONCLUSIONS}

The transverse stability of coasting beams has been studied under general assumptions on the shape of the working line (tune versus momentum) and on the momentum distribution function. The impedance model includes space-charge, resistive, and broad-band impedance contributions for an on-axis and an offaxis beam. The effect of space-charge distortion of the working line has been investigated. If the space-charge field decreases the local chromaticity at the coherent tune location, then its effect is determinant and must be compensated. If, on the contrary, the space charge field increases the local chromaticity, then its effect is to raise the threshold of the instability. The number and strength of multipoles needed to control the frequency distribution function has been studied. Pairs of consecutive even and odd multipoles are most effective in stabilizing the beam, whereas odd multipoles alone have a negative effect on the stability conditions. The most critical condition occurs during stacking for an off axis beam. In this case, when the coherent tune falls at the edge of the momentum distribution closest to the chamber wall, high magnetic multipoles are beneficial, since they provide the required local chromaticity with a smaller overall tune spread in the beam. When the above condition for the location of the coherent tune is not satisfied (as in the critical case of an off-axis beam for which the coherent tune is negative), then increasing the number of magnetic multipoles does not help, unless one uses a very large number of them. Thus, the study shows the importance of providing correcting elements for an asymetric condition.

\section{ACKNOWLEDGMENTS}

The authors wish to thank G. Parzen for discussions and information concerning the space charge pertaining in ISABELLE.- 


\author{
APPENDIX A \\ Coherent and Incoherent Space-Charge Tune Shift in ISABELLE
}

The vertical coherent tune shift due to direct space-charge forces, for a Gaussian space-charge distribution in both dimensions, is given by

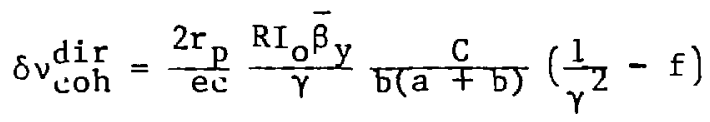

with

$$
\begin{aligned}
r_{p}= & \text { classical proton radius, } \\
\mathrm{c}= & \text { speed of light, } \\
\mathrm{e}= & \text { proton charge, } \\
\mathrm{R}= & \text { accelerator mean radius }=610 \mathrm{~m}, \\
\mathrm{I}_{\mathrm{O}}= & \text { beam current (peak current for a bunched beam), } \\
\mathrm{a}, \mathrm{b}= & \text { horizontal and vertical } 2 \text { times the rms beam } \\
& \text { dimensions, } \\
\bar{\beta}_{\mathrm{y}}= & \text { average vertical } \beta \text {-function } \sim 30 \mathrm{~m}, \\
\mathrm{C}= & \text { fraction of ring circumference where the formula (Al) } \\
\mathrm{f}= & \text { applies, and }
\end{aligned}
$$

In the insertion region, where the dispersion is zero, the assumption of a Gaussian charge distribution is satisfactory. In ISABELLE $\mathrm{C} \sim 0.3$ and if we assume

$$
\mathrm{a} \sim \mathrm{b}=\frac{2}{2.5} \sqrt{\mathrm{E}_{\mathrm{T}} \beta_{\mathrm{y}}},
$$

where $E_{t}$ is the transverse emittance (assumed the same in the horizontal and vertical planes) $=0.5 \pi \times 10^{-6} \mathrm{~m} \times \mathrm{rad}$ at $30 \mathrm{GeV}$. The 2.5 factor in the denominator of the beam size expression takes into account that the transverse emittances are defined for $95 \%$ of the particles in phase space.

With the above assumptions Eq. (Al) gives, for an unneutralized beam $(f=0)$,

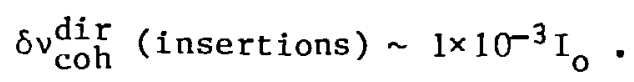

In the regular cells the application of Eq. (A1) is less valid, since the horizontal space-charge distribution is approximately flat with tails, rather than Gaussian. To obtain an order of magnitude estimate we still use (A1), assuming for a the expression

$$
a=\frac{2}{2.5}\left[\left(\bar{x}_{\mathrm{p}} \varepsilon_{\mathrm{M}}\right)+\left(\mathrm{E}_{\mathrm{T}} \bar{\beta}_{\mathrm{X}}\right)^{1 / 2}\right]
$$

with

$$
\begin{aligned}
& \overline{\mathrm{X}}_{\mathrm{p}}=\text { average dispersion } \sim 2.2 \mathrm{~m}, \\
& \vec{\beta}_{\mathrm{X}}=\text { average horizontal } \beta \text {-function } \sim 30 \mathrm{~m}, \text { and } \\
& \varepsilon_{M}=\text { momentum spread half-width. }
\end{aligned}
$$


At $30 \mathrm{GeV}$ we find, for a full stack, $\varepsilon_{M}=0.005, \delta \nu_{\mathrm{coh}}^{\mathrm{dir}}$ (regular cells) = $0.66 \times 10^{-3} \mathrm{I}_{0}$. The space-charge tune spread in the beam is approximately half the value of the coherent tune shift contributions of the regular cells, and we have assumed it to be of the octupole type. We have also assumed that this spread is independent of the width of the stack, i.e., that the ratio $I_{0} / a$ in Eq. ( $\mathrm{Al}$ ) to be constant, and $\mathrm{a} \gg \mathrm{b}$. In summary, we have used the following expressions for the vertical coherent and incoherent space-charge tune shifts, at $30 \mathrm{GeV}$ and without neutralization ( $f=0$ ):

$$
\begin{aligned}
& \delta v_{\text {coh }}=1.6 \times 10^{-3} \mathrm{I}_{\mathrm{o}} \\
& \delta v_{\text {inc }}=-1.6 \times 10^{-3} \mathrm{I}_{\mathrm{o}}+3 \times 10^{-3}\left(\frac{\varepsilon-\varepsilon_{\mathrm{d}}}{\varepsilon_{M}}\right)^{2}
\end{aligned}
$$

with

$\varepsilon=$ momentum deviation from the central orbit momentum, and $\varepsilon_{\mathrm{d}}=$ average momentum deviation for a displaced beam.

A detailed integration of the space charge field around the ISABELLE lattice, with realistic distribution functions, has been performed, 7 giving results in good agreement with our estimates. The results of the numerical integration show that the coherent horizontal tune shift is $\sim 35 \%$ smaller than the vertical one, while the octupolar space-charge tune spread is about half.

The effect of neutralization of the proton beam due to trapped electrons is important at high energy, since $\mathrm{f}$ must be $\geqslant 1 / \gamma^{2}$. At $400 \mathrm{GeV}$, assuming adiabatic debunching for the coasting beam (smallest momentum spread), we have

and

$$
\varepsilon_{M}=3.7 \times 10^{-4}
$$

$$
\varepsilon_{\mathrm{T}}=3.5 \pi \times 10^{-8} \mathrm{~m} \times \mathrm{rad} \text {. }
$$

We find, applying Eq. (Al),

$$
\delta v_{\text {coh }}=-2.3 \mathrm{f} \mathrm{I} \text { o }
$$

and for the incoherent tune shift, assuming an octupolar type of tune spread,

$$
\delta v_{\text {inc }}=2.3 f \mathrm{I}_{\mathrm{o}}-0.37 \mathrm{f} \mathrm{I}_{\mathrm{o}}\left(\frac{\varepsilon}{\varepsilon_{\mathrm{M}}}\right)^{2} \text {. }
$$

Again, detailed tracking of the space-charge field along the ISABELLE lattice agrees with our estimate. ${ }^{7}$ The tracking also shows that the horizontal linear tune shift is about half the vertical one, but the horizontal spread increases by about $60 \%$ in the horizontal plane.

7. G. Parzen, private comnunication (1982). 
The indirect space-charge field, i.e., the field created by image charges in the vacuum shamber, is small compared with the direct field in ISABELLE, and can be neglected even when the beam is closer to the wall during the stacking process. This has been confirmed by detailed tracking computations. 7 llere we shall use a qualitative argument to prove it.

The coherent vertical tune shift due to image charges for a line charge at a distance $x_{1}$ from the axis of a circular chamber of radius $h$ is given by ${ }^{6}$

$$
\delta v_{\operatorname{coh}}^{\text {ind }} \simeq \frac{2 r_{p}}{e c} \frac{R I_{o}}{\gamma} \bar{\beta}_{y} \frac{x_{1}^{2}}{\left(h^{2}-x_{1}^{2}\right)^{2}}
$$

so that the ratio between direct and indirect tune shifts is, from Eq. (Al)

$$
\left(\frac{\delta v^{\text {dir }}}{\delta v^{\text {ind }}}\right)_{c o h} \sim \frac{1}{b(a+b)} \frac{\left(h^{2}-x_{1}^{2}\right)^{2}}{x_{1}^{2}}
$$

For a $20 \%$ stacked beam, the ratio is about 170 (with $\mathrm{h}=4.4 \mathrm{~cm}$ ). The same qualitative argument applies to the incoherent tune shift and spread, both horizontal and vertical. 


\section{APPENDIX B \\ Transverse Space-Charge Distributton Function}

1. Coasting beam distribution

We have assumed a distribution which is flat in the central part of the beam. At the tails the charge density decays like a biquar.catic function (Figure 7)

$$
\begin{aligned}
& g_{0}(\varepsilon)=g_{1}, \text { for }-\varepsilon, \leqslant \varepsilon \leqslant \varepsilon_{1} ; \\
& g_{0}(\varepsilon)=g_{1}\left[1-\left(\frac{\varepsilon-\varepsilon_{1}}{\varepsilon_{M}-\varepsilon_{1}}\right)^{2}\right]^{2} .
\end{aligned}
$$

$g_{1}$ is determined by the normalization condition

$$
\int_{-W}^{W} g_{0}(\varepsilon) d \varepsilon=N
$$

where $\mathrm{N}$ is the longitudinal charge density; after solving (B2) with the function described by $(B 1)$ we find

$$
g_{1}=7.5 N /\left(7 \varepsilon_{1}+8 \varepsilon_{M}\right) .
$$

2. Bunched beam distributions

a) Elliptic distribution (Figure 7), after normalization:

$$
g_{0}(\varepsilon)=\frac{2 N}{\varepsilon_{M}{ }^{\pi}}\left[1-\left(\frac{\varepsilon}{\varepsilon_{M}}\right)^{2}\right]
$$

b) Parabolic distribution (Figure 7):

$$
g_{o}(\varepsilon)=\frac{3 N}{4 \varepsilon_{M}}\left[1-\left(\frac{\varepsilon}{\varepsilon_{M}}\right)^{2}\right] \text {. }
$$

Figure 7 shows that a parabolic distribution has sharper edges than an elliptical one. 
APPENDIX C

Impedance of an off-Axis Beam

When the center of the charge of the beam is off-axis, as during the stacking process, then Eqs. (17) and (18) of Part I do not apply. The spacecharge term is unchanged (since, as we have shown in Appendix A, the indirect effect of the image charges is negligible compared with the direct charge effect), but the resistance and broad-band impedance term change because of the proximity of the wall.

The expression of the resistance wall impedance has been calculated ${ }^{8}$ for an off-axis beam. For a pencil beam it is

$$
z_{\perp}\left(\text { resistive wa11) }=-(1+i) \frac{\delta(\omega)}{b^{3}} \frac{b^{4}\left(b^{2}+x_{0}^{2}\right)}{\left(b^{2}-x_{0}^{2}\right)^{3}}\right.
$$

where $b$ is the beam pipe radius and $x_{0}$ the displacement of the beam from the axis of the chamber.

As far as the broad-band impedance is concerned, we have used the expression (18) of Part I, replacing the chamber radius b with an "equivalent" radius $b-x_{0}$. The resultant impedance as a function of mode number is shown in Figure 1 .

8. E.D. Courant and M. Month, Transverse Resistance Wall Instability of an Off-Axis Ribbon Beam in a Circular Chamber, BNL-50875, June 1978. 